\title{
Generalized Laplace-Type Transform Method for Solving Multilayer Diffusion Problems
}

\author{
Mohamed Akel $\mathbb{D},{ }^{1,2}$ Hillal M. Elshehabey $\mathbb{D}^{1,2}$ and Ragaa Ahmed $\mathbb{D}^{1,2}$ \\ ${ }^{1}$ Mathematics Department, Faculty of Science, South Valley University, Qena 83523, Egypt \\ ${ }^{2}$ Academy of Scientific Research and Technology (ASRT), 101 Kasr Al-Ainy St., Cairo 11516, Egypt \\ Correspondence should be addressed to Mohamed Akel; makel@sci.svu.edu.eg
}

Received 8 October 2021; Accepted 27 November 2021; Published 7 March 2022

Academic Editor: Lars E. Persson

Copyright (c) 2022 Mohamed Akel et al. This is an open access article distributed under the Creative Commons Attribution License, which permits unrestricted use, distribution, and reproduction in any medium, provided the original work is properly cited.

\begin{abstract}
Multilayer diffusion problems have found significant importance that they arise in many medical, environmental, and industrial applications of heat and mass transfer. In this article, we study the solvability of a one-dimensional nonhomogeneous multilayer diffusion problem. A new generalized Laplace-type integral transform is used, namely, the $\mathbb{M}_{\rho, m}$-transform. First, we reduce the nonhomogeneous multilayer diffusion problem into a sequence of one-layer diffusion problems including time-varying given functions, followed by solving a general nonhomogeneous one-layer diffusion problem via the $\mathbb{M}_{\rho, m}$-transform. Hence, by means of general interface conditions, a renewal equations' system is determined. Finally, the $\mathbb{M}_{\rho, m}$-transform and its analytic inverse are used to obtain an explicit solution to the renewal equations' system. Our results are of general attractiveness and comprise a number of previous works as special cases.
\end{abstract}

\section{Introduction}

The multilayer diffusion problems are typical models for a variety of solute transport phenomena in layered permeable media, such as advection, dispersion, and reaction diffusions [1-10]. These problems have had their importance due to their natural prevalence in a remarkably large number of applications such as chamber-based gas flux measurements [11], contamination and decontamination in permeable media $[6,12]$, drug-eluting stent $[13,14]$, drug absorption $[15,16]$, moisture propagation in woven fabric composites [17], permeability of the skin [18], and wool-washing [19]. Further applications have been considered in [20,21].

As epidemiological models, reaction-diffusion problems are widely used to model and analyze the spread of diseases such as the global COVID-19 pandemic caused by SARSCoV-2. These models describe the spatiotemporal prevalence of the viral pandemic and apprehend the dynamics depending on human habits and geographical features. The models estimate a qualitative harmony between the simulated prediction of the local spatiotemporal spread of a pan- demic and the epidemiological collected datum (see [22, 23]). These data-driven emulations can essentially inform the respective authorities to purpose efficient pandemicarresting measures and foresee the geographical distribution of vital medical resources. Moreover, such studies explore alternate scenarios for the repose of lockdown restrictions based on the local inhabitance densities and the qualitative dynamics of the infection. For more applications, one can refer, e.g., to $[24,25]$.

Although the numerical methods are usually applied to solve the diffusion problems, especially in the heterogeneous permeable media, the analytic solutions, when available, are characterized by their exactness and continuity in space and time. In the context of obtaining numerical solutions for such models, we refer to the following references [26-30]. In this work, we focus on analytic solutions of certain nonhomogeneous diffusion problems in multilayer permeable media. Here, the retardation factors are assumed to be constant, the dispersion coefficients vary across layers, but being constants within each layer, and the free terms are (arbitrary) time-varying functions. 
Analytic and semianalytic solutions of multilayer diffusion problems are developed by using the integral transforms [6, 25, 31-40]. Applying Laplace transforms, to solve multilayer diffusion problems, has advantages as an applicable tool in handling different types of boundary conditions and averts solving complicated transcendental equations as demanded by eigenfunction expansion methods. Further works involving the Laplace transform have studied the permeable layered reaction diffusion problem in $[41,42]$. Solutions obtained in these works are restricted to two layers as well as obtaining the inverse Laplace transform numerically. In the same context, generalized integral transform techniques, for short GITT, are well-established hybrid approaches for solving diffusion and convection-diffusion problems, in which hybrid refers to the combination of classical analytical methods with modern computational tools aimed at accurate, robust, and low-cost solutions [43-47]. In the current work, we aim to extend, generalize, and merge results in [31, 33, $38,40,42]$ to solve certain nonhomogeneous diffusion problems in one-dimensional $n$-layered media. We use a new generalized integral transform recently introduced in [48]. The obtained solutions are applicable to more general linear nonhomogeneous diffusion equations, finite media consisting of arbitrary many layers, continuity and dispersive flow at the contact interfaces between sequal layers, and transitory boundary conditions of the arbitrary type at the inlet and outlet. To the best knowledge of the authors, analytical solutions verifying all the above mentioned conditions have not been previously reported in the literature which strongly motivates this current work.

In the remaining part of this introductory section, in Subsection 1.1, the multilayer diffusion problem is described, and then, it is reformulated as a sequence of one-layer diffusion problems having boundary conditions including given time-depending functions. Basic properties for the $\mathbb{M}_{\rho, m}$-transform that will be needed in this work are stated in Subsection 1.2. The remaining sections are constructed as follows: in Section 2, we discuss the solvability of a general linear nonhomogeneous one-layer diffusion problem with arbitrary time-varying data, using the $\mathbb{M}_{\rho, m}$-transform. Section 3 is devoted to our main multilayer diffusion problem, where in Subsection 3.1, we solve a two-layer problem to shed light on the basic idea by considering this simple case. Further, in Subsection 3.2, we return to benefit from the results obtained in Section 2 and Subsection 3.1 to solve the main multilayer diffusion problems (2)-(8) (see Subsection 1.1 below).

1.1. Mathematical Modeling for Nonhomogeneous n-Layer Diffusion Systems. A one-dimensional diffusion problem in an $n$-layered permeable medium is set out as follows. Let

$$
\alpha=x_{0}<x_{1}<\cdots<x_{n-1}<x_{n}=\beta,
$$

be a finite partition of the interval $[\alpha, \beta]$. In each subinterval $\left[x_{j-1}, x_{j}\right]$, with $j=1,2, \cdots, n$, the component function $\varphi_{j}(x, t)$ satisfies the partial differential equation (PDE)

$$
\frac{\partial \varphi_{j}}{\partial t}=d_{j} \frac{\partial^{2} \varphi_{j}}{\partial x^{2}}+\lambda(t, \tau) r_{j}(x, t), x \in\left(x_{j-1}, x_{j}\right), \quad t, \tau>0,
$$

where $d_{j} \geq 0$, for all $1 \leq j \leq n$, are the diffusion coefficients and

$$
\lambda(t, \tau)=\left(\frac{t^{m}}{\tau^{m}}+\tau^{m}\right)^{-\rho}, \quad t \geq 0, \tau>0
$$

with $m \in \mathbb{Z}_{+}=\{1,2,3, \cdots\}, \rho \in \mathbb{C}, \operatorname{Re}(\rho)>0$. Here, the function-term $\lambda(t, \tau) r_{j}(x, t)$ physically means the external source term that could be applied to the diffusion equation with $r_{j}(x, t)$ depends on time and space while the other factor of the source term, i.e., $\lambda$, depends only on time. This last factor could be, for instance, a periodic-time magnetic source.

The initial conditions (ICs) are assumed as

$$
\varphi_{j}(x, 0)=\eta_{j}(x), x \in\left[x_{j-1}, x_{j}\right], 1 \leq j \leq n .
$$

The boundary conditions (BCs) are posited as

(i) The outer BCs (at the inlet $x=\alpha$ and the outlet $x=\beta$ ) are general Robin boundary conditions as

$$
\begin{array}{r}
\iota \varphi_{1}(\alpha, t)+\iota \frac{\partial \varphi_{1}}{\partial x}(\alpha, t)=\lambda(t, \tau) \zeta(t), \\
\ell \varphi_{n}(\beta, t)+l \frac{\partial \varphi_{n}}{\partial x}(\beta, t)=\lambda(t, \tau) \xi(t),
\end{array}
$$

for all $t \geq 0$, with $\imath, \iota, \ell$, and $l$ are constants satisfying $|\imath|$ $+|\iota|>0,|\ell|+|l|>0$.

(ii) The inner BCs (the interface conditions) are

$$
\begin{gathered}
\varphi_{j}\left(x_{j}, t\right)=\Lambda_{j} \varphi_{j+1}\left(x_{j}, t\right), \\
v_{j} \varphi_{j}\left(x_{j}, t\right)+\mu_{j} \frac{\partial \varphi_{j}}{\partial x}\left(x_{j}, t\right)=v_{j+1} \varphi_{j+1}\left(x_{j}, t\right)+\mu_{j+1} \frac{\partial \varphi_{j+1}}{\partial x}\left(x_{j}, t\right),
\end{gathered}
$$

for all $t \geq 0$, with $\left|v_{j}\right|+\left|\mu_{j}\right|>0$ for all $j=1,2, \cdots, n-1$.

For appropriate given functions $\eta_{1}, \cdots, \eta_{n}, \zeta$, and $\xi$, we are going to find an analytic solution of the problems (2)-(8) using the $\mathbb{M}_{\rho, m}$-generalized integral transform, introduced recently in [48]. Problems (2)-(8) can be reduced into the following sequence of one-layer diffusion problems. 
(i) In the inlet layer, i.e., $x \in\left[x_{0}, x_{1}\right]$,

$$
\begin{gathered}
\frac{\partial \varphi_{1}}{\partial t}=d_{1} \frac{\partial^{2} \varphi_{1}}{\partial x^{2}}+\lambda(t, \tau) r_{1}(x, t), \quad x \in\left(x_{0}, x_{1}\right), t, \tau>0, \\
\varphi_{1}(x, 0)=\eta_{1}(x), \quad x \in\left[x_{0}, x_{1}\right], \\
\iota \varphi_{1}\left(x_{0}, t\right)+\iota \frac{\partial \varphi_{1}}{\partial x}\left(x_{0}, t\right)=\lambda(t, \tau) \zeta_{1}(t), \quad t \geq 0, \tau>0, \\
\nu_{1} \varphi_{1}\left(x_{1}, t\right)+\mu_{1} \frac{\partial \varphi_{1}}{\partial x}\left(x_{1}, t\right)=\lambda(t, \tau) \xi_{1}(t), \quad t \geq 0, \tau>0 .
\end{gathered}
$$

(ii) In the interior layers, i.e., $x \in\left[x_{j-1}, x_{j}\right], 2 \leq j \leq n-1$,

$$
\begin{gathered}
\frac{\partial \varphi_{j}}{\partial t}=d_{j} \frac{\partial^{2} \varphi_{j}}{\partial x^{2}}+\lambda(t, \tau) r_{j}(x, t), \quad x \in\left(x_{j-1}, x_{j}\right), t, \tau>0, \\
\varphi_{j}(x, 0)=\eta_{j}(x), \quad x \in\left[x_{j-1}, x_{j}\right], \\
v_{j} \varphi_{j}\left(x_{j-1}, t\right)+\mu_{j} \frac{\partial \varphi_{j}}{\partial x}\left(x_{j-1}, t\right)=\lambda(t, \tau) \zeta_{j}(t), \quad t \geq 0, \tau>0, \\
v_{j} \varphi_{j}\left(x_{j}, t\right)+\mu_{j} \frac{\partial \varphi_{j}}{\partial x}\left(x_{j}, t\right)=\lambda(t, \tau) \xi_{j}(t), \quad t \geq 0, \tau>0 .
\end{gathered}
$$

(iii) In the outlet layer, i.e., $x \in\left[x_{n-1}, x_{n}\right]$,

$$
\begin{gathered}
\frac{\partial \varphi_{n}}{\partial t}=d_{n} \frac{\partial^{2} \varphi_{n}}{\partial x^{2}}+\lambda(t, \tau) r_{n}(x, t), \quad x \in\left(x_{n-1}, x_{n}\right), t, \tau>0, \\
\varphi_{n}(x, 0)=\eta_{n}(x), \quad x \in\left[x_{n-1}, x_{n}\right], \\
v_{n} \varphi_{n}\left(x_{n-1}, t\right)+\mu_{n} \frac{\partial \varphi_{n}}{\partial x}\left(x_{n-1}, t\right)=\lambda(t, \tau) \zeta_{n}(t), \quad t \geq 0, \tau>0, \\
\ell \varphi_{n}\left(x_{n}, t\right)+l \frac{\partial \varphi_{n}}{\partial x}\left(x_{n}, t\right)=\lambda(t, \tau) \xi_{n}(t), \quad t \geq 0, \tau>0 .
\end{gathered}
$$

Remark 1. Each of the initial boundary value problems (9)-(11) is a case of the one-layer nonhomogeneous diffusion problem that will be discussed in Section 2 below.

Now, in view of the inner boundary conditions (7) and (8), the time-varying functions $\zeta_{j}$ and $\xi_{j}$ for all $2 \leq j \leq n$ are subject to

$$
\zeta_{j}(t)=\xi_{j-1}(t), \quad 2 \leq j \leq n,
$$

so that

$$
\begin{aligned}
& \zeta_{j}(t)= \begin{cases}\zeta(t), & j=1, \\
\xi_{j-1}(t), & 2 \leq j \leq n,\end{cases} \\
& \xi_{j}(t)= \begin{cases}\left(v_{j} \varphi_{j}\left(x_{j}, t\right)+\mu_{j} \frac{\partial \varphi_{j}}{\partial x}\left(x_{j}, t\right)\right) \lambda^{-1}(t, \tau), & 1 \leq j \leq n-1, \\
\xi(t), & j=n .\end{cases}
\end{aligned}
$$

While the outer boundary data $\zeta_{1}(t)=\zeta(t)$ and $\xi_{n}(t)=$ $\xi(t)$ are given in (5) and (6), respectively, the functions $\zeta_{j}($ $2 \leq j \leq n)$ can be determined once we specify the functions $\xi_{j}(1 \leq j \leq n-1)$. Hence, we have to find $\xi_{j}, 1 \leq j \leq n-1$. To do so, we should use the first matching condition (7).

1.2. Srivastava-Luo-Raina Generalized Integral Transform. In [48], Srivastava et al. introduced the following generalized integral transform:

$$
\mathbb{M}_{\rho, m}[\varphi(t)](s, \tau)=\int_{0}^{\infty} \frac{e^{-s t} \varphi(\tau t)}{\left(t^{m}+\tau^{m}\right)^{\rho}} d t
$$

for a continuous (or piecewise continuous) function $\varphi$ on [ $0, \infty)$, where $\rho \in \mathbb{C} ; \operatorname{Re}(\rho) \geq 0 ; m \in \mathbb{Z}_{+}, s>0$ is the transform variable and $\tau>0$ is a parameter. The basic properties of the $\mathbb{M}_{\rho, m}$-transform are given in [48]. Next, we recall some of these properties, which are needed in the present work. Indeed, as introduced in [48] the $\mathbb{M}_{\rho, m}$-transform is closely related with the well-known integral transforms, the Laplace, natural, and Sumudu transforms. The Laplace transform is defined by

$$
\mathbb{L}[\varphi(t)](s)=\int_{0}^{\infty} e^{-s t} \varphi(t) d t, \operatorname{Re}(s)>0 .
$$

So, from (14) and (15), we have the following duality relations:

$$
\begin{gathered}
\mathbb{L}[\varphi(t)](s)=\mathbb{M}_{0, m}[\varphi(t)](s, 1), \quad \operatorname{Re}(s)>0, \\
\mathbb{M}_{\rho, m}[\varphi(t)](s, \tau)=\mathbb{L}\left[\frac{\varphi(\tau t)}{\left(t^{m}+\tau^{m}\right)^{\rho}}\right](s), \quad s, \tau>0, \\
\mathbb{M}_{\rho, m}[\varphi(t)](s, \tau)=\frac{1}{\tau} \mathbb{L}\left[\frac{\varphi(t)}{\left(\left(t^{m} / \tau^{m}\right)+\tau^{m}\right)^{\rho}}\right]\left(\frac{s}{\tau}\right), \quad s, \tau>0, \\
\mathbb{M}_{\rho, m}\left[\left(\frac{t^{m}}{\tau^{m}}+\tau^{m}\right)^{\rho} \varphi(t)\right](s, \tau)=\mathbb{L}[\varphi(\tau t)](s), \quad s, \tau>0 .
\end{gathered}
$$

Setting $\rho=0$ in (14), we recover the natural transform defined as (see $[49,50]$ )

$$
\mathbb{N}[\varphi(t)](s, \tau)=\int_{0}^{\infty} e^{-s t} \varphi(\tau t) d t, \quad s>0, \tau>0 .
$$


Thus, we have the following $\mathbb{M}_{\rho, m}-\mathbb{N}$-transform duality

$$
\begin{gathered}
\mathbb{N}[\varphi(t)](s, \tau)=\mathbb{M}_{0, m}[\varphi(t)](s, \tau), \\
\mathbb{M}_{\rho, m}[\varphi(t)](s, \tau)=\mathbb{N}\left[\frac{\varphi(t)}{\left(\left(t^{m} / \tau^{m}\right)+\tau^{m}\right)^{\rho}}\right](s, \tau), \quad s>0, \tau>0,
\end{gathered}
$$

$$
\mathbb{M}_{\rho, m}\left[\left(\frac{t^{m}}{\tau^{m}}+\tau^{m}\right)^{\rho} \varphi(t)\right](s, \tau)=\mathbb{N}[\varphi(t)](s, \tau) ; \quad s>0, \tau>0 .
$$

The Sumudu transform is defined by [51-53]

$$
\mathbb{S}[\varphi(t)](\tau)=\int_{0}^{\infty} e^{-t} \varphi(\tau t) d t, \quad \tau>0
$$

Thus,

$$
\begin{gathered}
\mathbb{S}[\varphi(t)](\tau)=\mathbb{M}_{0, m}[\varphi(t)](0, \tau), \quad \tau>0, \\
\mathbb{M}_{\rho, m}[\varphi(t)](s, \tau)=\frac{1}{s} \mathbb{S}\left[\frac{\varphi(t)}{\left(t^{m} / \tau^{m}+\tau^{m}\right)^{\rho}}\right]\left(\begin{array}{c}
\tau \\
s
\end{array}\right), \quad s, \tau>0 .
\end{gathered}
$$

Based on these dualities of the $\mathbb{M}_{\rho, m}$-transform (14) and these well-known integral transforms, it seems to be interesting to apply the $\mathbb{M}_{\rho, m}$-transform (14) in solving a variety of boundary and initial-boundary value problems. In this context, we recall the following results [48]:

(i) Let $\varphi^{(n)}(t)$ be the $n^{\text {th }}$-order $t$-derivative of the function $\varphi(t)$ and $|\varphi(t)| \leq K e^{t / \gamma}$ with $K>0, \gamma>0$. Then,

$$
\begin{aligned}
\mathbb{M}_{\rho, m} & {\left[\left(\frac{t^{m}}{\tau^{m}}+\tau^{m}\right)^{\rho} \varphi^{(n)}(t)\right](s, \tau)=\frac{s^{n}}{\tau^{n}} \mathbb{N}[\varphi(t)](s, \tau) } \\
& -\sum_{k=0}^{n-1} \frac{s^{k}}{\tau^{k+1}} \varphi^{(n-k-1)}(0),
\end{aligned}
$$

where $\mathbb{N}[\varphi(t)](s, \tau)$ is defined by (18). Using the duality (21) in (25), we find

$$
\begin{aligned}
\mathbb{N} & {\left[\varphi^{(n)}(t)\right](s, \tau)=\frac{s^{n}}{\tau^{n}} \mathbb{N}[\varphi(t)](s, \tau) } \\
& -\sum_{k=0}^{n-1} \frac{s^{k}}{\tau^{k+1}} \varphi^{(n-k-1)}(0), \quad n=0,1, \cdots
\end{aligned}
$$

(ii) Again, using the dualities stated before a convolution formula for the $\mathbb{M}_{\rho, m}$-transform (14) can be obtained as follows. Here, the convolution for the Laplace transform will be considered; that is, for the functions $\varphi$ and $\psi$, the convolution formula is given as

$$
(\varphi * \psi)(t)=\int_{0}^{t} \varphi(x) \psi(t-x) d x=\int_{0}^{t} \varphi(t-x) \psi(x) d x .
$$

$\tau)$,

If $\Phi(s, \tau)=\mathbb{M}_{\rho, m}[\varphi(t)](s, \tau)$ and $\Psi(s, \tau)=\mathbb{M}_{\rho, m}[\psi(t)](s$, then-

$\tau \Phi(s, \tau) \Psi(s, \tau)=\tau \int_{0}^{\infty}\left(e^{-s t_{1}} \varphi\left(\tau t_{1}\right) /\left(t_{1}^{m}+\tau^{m}\right)^{\rho}\right) d t_{1} \int_{0}^{\infty}\left(e^{-s t_{2}} \psi(\right.$ $\left.\left.\tau t_{2}\right) /\left(t_{2}^{m}+\tau^{m}\right)^{\rho}\right) d t_{2}=\tau \int_{0}^{\infty} \int_{0}^{\infty} e^{-s\left(t_{1}+t_{2}\right)} \tilde{\varphi}\left(\tau t_{1}\right) \tilde{\psi}\left(\tau t_{2}\right) d t_{1} d t_{2}$, where

$$
\tilde{\varphi}(t)=\frac{\varphi(t)}{\left(t^{m} / \tau^{m}+\tau^{m}\right)^{\rho}}, \tilde{\psi}(t)=\frac{\psi(t)}{\left(t^{m} / \tau^{m}+\tau^{m}\right)^{\rho}} .
$$

Setting $t_{1}+t_{2}=t$ in the last equality, one gets

$$
\begin{aligned}
\tau \Phi(s, \tau) \Psi(s, \tau) & =\tau \int_{0}^{\infty} \int_{t_{2}}^{\infty} e^{-s t} \tilde{\varphi}\left(\tau\left(t-t_{2}\right)\right) \tilde{\psi}\left(\tau t_{2}\right) d t d t_{2} \\
& =\tau \int_{0}^{\infty} e^{-s t} d t \int_{0}^{t} \tilde{\varphi}\left(\tau\left(t-t_{2}\right)\right) \tilde{\psi}\left(\tau t_{2}\right) d t_{2} .
\end{aligned}
$$

Here, changing of the integral order is used. Thus, using the duality of the $\mathbb{M}_{\rho, m}$ and $\mathbb{N}$ transforms (see (20)), we find

$$
\tau \Phi(s, \tau) \Psi(s, \tau)=\mathbb{N}[\tilde{\varphi} * \tilde{\psi}](s, \tau) .
$$

Remark 2. If we put $\rho=0$ in (30), the case being interesting later in our work, then we get

$$
\tau \mathbb{N}[\varphi(t)](s, \tau) \mathbb{N}[\psi(t)](s, \tau)=\mathbb{N}[(\varphi * \psi)(t)](s, \tau) .
$$

(iii) Once again, ... again, using the dualities stated before an inversion formula of the $\mathbb{M}_{\rho, m}$-transform (14) is given (see Theorem 4.1 of [48]) as

$$
\begin{aligned}
\varphi(t) & =\left(\frac{t^{m}}{\tau^{m}}+\tau^{m}\right)^{\rho} \mathbb{L}^{-1}\left\{\mathbb{M}_{\rho, m}[\varphi(t)](s, t)\right\}\left(\frac{t}{\tau}\right) \\
& =\frac{1}{2 \pi i}\left(\frac{t^{m}}{\tau^{m}}+\tau^{m}\right)^{\rho} \int_{c-i \infty}^{c+i \infty} e^{s t / \tau} \mathbb{M}_{\rho, m}[\varphi(t)(s, \tau)] d s, \quad c, \tau>0,
\end{aligned}
$$

as long as the integral converges absolutely. In case, when $\rho=0$ one obtains the following inversion formula of the natural transform (see Theorem 5.3 of [49])

$$
\varphi(t)=\frac{1}{2 \pi i} \int_{c-i \infty}^{c+i \infty} e^{s t / \tau} \mathbb{N}[\varphi(t)](s, \tau) d s, \quad c, \tau>0 .
$$

The residue theorem (see, e.g., [54]) is usually used to calculate the contour integrals in (32) and (33). 


\section{One-Layer Nonhomogeneous Diffusion System}

Now, we investigate the solvability for the following onelayer nonhomogeneous initial boundary value problem:

$$
\begin{gathered}
\frac{\partial \varphi}{\partial t}=d \frac{\partial^{2} \varphi}{\partial x^{2}}+\lambda(t, \tau) r(x, t), \quad x \in(\alpha, \beta), \quad t, \tau>0, \\
\varphi(x, 0)=\eta(x), \quad x \in[\alpha, \beta], \\
\iota \varphi(\alpha, t)+\iota \frac{\partial \varphi}{\partial x}(\alpha, t)=\lambda(t, \tau) \zeta(t), \quad t \geq 0, \tau>0, \\
\ell \varphi(\beta, t)+l \frac{\partial \varphi}{\partial x}(\beta, t)=\lambda(t, \tau) \xi(t), \quad t \geq 0, \tau>0,
\end{gathered}
$$

where $d, \imath, \imath, \ell$, and $l$ are constants such that $|\imath|+|\iota|>0,|\ell|$ $+|l|>0$, and $\lambda, r, \eta, \zeta$, and $\xi$ are given functions with $\lambda$ as in (3).

Applying the $\mathbb{M}_{\rho, m}$-transform defined by (14), to (34), yields

$\mathbb{M}_{\rho, m}\left[\frac{1}{\lambda(t, \tau)} \varphi_{t}(x, t)\right]=d \mathbb{M}_{\rho, m}\left[\frac{1}{\lambda(t, \tau)} \varphi_{x x}(x, t)\right]+\mathbb{M}_{\rho, m}[r(x, t)]$.

Using the duality of the $\mathbb{M}_{\rho, m}$-transform and the natural transform given by (21) and (25), Equation (38) can be reduced to

$$
\begin{aligned}
\frac{s}{\tau d} & \mathbb{N}[\varphi(x, t)](x ; s, \tau)-\mathbb{N}\left[\varphi_{x x}(x, t)\right](x ; s, \tau)-\frac{1}{\tau d} \varphi(x, 0) \\
& =\frac{1}{d} \mathbb{N}[\lambda(t, \tau) r(x, t)](x ; s, \tau),
\end{aligned}
$$

where $\mathbb{N}[\varphi(t)](s, \tau)$ is defined by (18). Setting

$$
\widehat{\varphi}(x ; s, \tau)=\mathbb{N}[\varphi(x, t)](x ; s, \tau),
$$

then, (39) can be expressed as

$$
\widehat{\varphi}_{x x}(x ; s, \tau)-\frac{s}{\tau d} \widehat{\varphi}(x ; s, \tau)=F(x ; s, \tau),
$$

where

$$
F(x ; s, \tau)=-\frac{1}{d} \mathbb{N}[\lambda(t, \tau) r(x, t)](x ; s, \tau)-\frac{1}{\tau d} \eta(x) .
$$

Applying the variation of the parameter method to the nonhomogeneous equation (41) gives the general solution as

$$
\begin{aligned}
\widehat{\varphi}(x ; s, \tau)= & A \cosh \sqrt{\frac{s}{\tau d}} x+B \sinh \sqrt{\frac{s}{\tau d}} x \\
& +\sqrt{\frac{\tau d}{s}} \int_{\alpha}^{x} F(y ; s, \tau) \sinh \sqrt{\frac{s}{\tau d}}(x-y) d y,
\end{aligned}
$$

where $A$ and $B$ are arbitrary invariants which can depend on $s$ and $\tau$. Differentiating (43) with respect to $x$, gives

$$
\begin{aligned}
\widehat{\varphi}_{x}(x ; s, \tau)= & A \sqrt{\frac{s}{\tau d}} \sinh \sqrt{\frac{s}{\tau d}} x+B \sqrt{\frac{s}{\tau d}} \cosh \sqrt{\frac{s}{\tau d}} x \\
& +\int_{\alpha}^{x} F(y ; s, \tau) \cosh \sqrt{\frac{s}{\tau d}}(x-y) d y .
\end{aligned}
$$

Transforming the boundary conditions (36) and (37), implies

$$
\begin{aligned}
& \imath \widehat{\varphi}(\alpha ; s, \tau)+\imath \widehat{\varphi}_{x}(\alpha ; s, \tau)=\mathbb{M}_{\rho, m}[\zeta(t)]=\mathbb{N}[\lambda(t, \tau) \zeta(t)] \\
& \ell \widehat{\varphi}(\beta, s, t)+l \widehat{\varphi}_{x}(\beta ; s, t)=\mathbb{M}_{\rho, m}[\xi(t)]=\mathbb{N}[\lambda(t, \tau) \xi(t)]
\end{aligned}
$$

For simplicity, we set the following vector notations:

$$
\mathfrak{a}=(l, \iota), \mathfrak{b}=(\ell, l)
$$

$$
\mathfrak{Q}(y ; s, \tau)=\left(\cosh \sqrt{\frac{s}{\tau d}} y, \sqrt{\frac{s}{\tau d}} \sinh \sqrt{\frac{s}{\tau d}} y\right),
$$

$$
\mathfrak{s}(y ; s, \tau)=\left(\sinh \sqrt{\frac{s}{\tau d}} y, \sqrt{\frac{s}{\tau d}} \cosh \sqrt{\frac{s}{\tau d}} y\right) .
$$

Obviously, we have

$$
\frac{\partial \mathfrak{Q}}{\partial y}(y ; s, \tau)=\sqrt{\frac{s}{\tau d}} \mathfrak{S}(y ; s, \tau), \frac{\partial \mathfrak{r}}{\partial y}(y ; s, \tau)=\sqrt{\frac{s}{\tau d}} \mathfrak{Q}(y ; s, \tau) .
$$

Substituting (43) and (44) into (45) and using the vector notation, we give the algebraic linear system

$$
\left(\begin{array}{cc}
\langle\mathfrak{a}, \mathfrak{L}(\alpha ; s, \tau)\rangle & \langle\mathfrak{a}, \mathfrak{S}(\alpha ; s, \tau)\rangle \\
\langle\mathfrak{b}, \mathfrak{Q}(\beta ; s, \tau)\rangle & \langle\mathfrak{b}, \mathfrak{c}(\beta ; s, \tau)\rangle
\end{array}\right)\left(\begin{array}{c}
A \\
B
\end{array}\right)=\left(\begin{array}{c}
\mathbb{G}(s, \tau) \\
H^{+}(x ; s, \tau)
\end{array}\right),
$$

where $\langle\cdot\rangle$ is the usual dot product in $\mathbb{R}^{2}$, and

$$
\mathbb{G}(s, \tau)=\mathbb{N}[\lambda(t, \tau) \zeta(t)]
$$

$$
H^{+}(x ; s, \tau)=H(s, \tau)-\sqrt{\frac{\tau d}{s}} \int_{\alpha}^{\beta} F(y ; s, \tau)\langle\mathfrak{b}, \mathfrak{c}(\beta-y ; s, \tau)\rangle d y,
$$

with $H(s, \tau)=\mathbb{N}[\lambda(t, \tau) \xi(t)]$ and $\zeta(t)$ and $\xi(t)$ are the boundary data given in (36) and (37), respectively. The 
solution $(A, B)$ of system $(50)$ is

$$
\begin{gathered}
A \Delta(s)=\mathbb{G}(s, \tau)\langle\mathfrak{b}, \mathfrak{c}(\beta ; s, \tau)\rangle-H^{+}(x ; s, \tau)\langle\mathfrak{a}, \mathfrak{c}(\alpha ; s, \tau)\rangle, \\
B \Delta(s)=-\left[\mathbb{G}(s, \tau)\langle\mathfrak{b}, \mathfrak{L}(\beta ; s, \tau)\rangle-H^{+}(x ; s, \tau)\langle\mathfrak{a}, \mathfrak{Q}(\alpha ; s, \tau)\rangle\right],
\end{gathered}
$$

where

$$
\Delta(s)=\langle\mathfrak{b}, \mathfrak{C}(\beta ; s, \tau)\rangle\langle\mathfrak{a}, \mathfrak{Q}(\alpha ; s, \tau)\rangle-\langle\mathfrak{a}, \mathfrak{C}(\alpha ; s, \tau)\rangle\langle\mathfrak{b}, \mathfrak{L}(\beta ; s, \tau)\rangle,
$$

is the determinant of the coefficient matrix of system (50). Substituting the constants $A$ and $B$ into (43) gives

$$
\begin{aligned}
\widehat{\varphi}(x ; s, \tau)= & \frac{\cosh \sqrt{(s / \tau d)} x}{\Delta(s)}(\mathbb{G}(s, \tau)\langle\mathfrak{b}, \mathfrak{S}(\beta ; s, \tau)\rangle \\
& \left.-H^{+}(x ; s, \tau)\langle\mathfrak{a}, \mathfrak{S}(\alpha ; s, \tau)\rangle\right) \\
& +\frac{\sinh \sqrt{(s / \tau d)} x}{\Delta(s)}(-\mathbb{G}(s, \tau)\langle\mathfrak{b}, \mathfrak{Q}(\beta ; s, \tau)\rangle \\
& \left.+H^{+}(x ; s, \tau)\langle\mathfrak{a}, \mathfrak{L}(\alpha ; s, \tau)\rangle\right) \\
& +\sqrt{\frac{\tau d}{s}} \int_{\alpha}^{x} \sinh \sqrt{\frac{s}{\tau d}}(x-y) F(y ; s, \tau) d y,
\end{aligned}
$$

which can be rewritten as

$$
\begin{aligned}
\widehat{\varphi}(x ; s, \tau)= & \frac{\mathbb{G}(s, \tau) \psi(x, \beta ; s, \tau, \mathfrak{b})}{\Delta(s)} \\
& -\frac{H(s, \tau) \psi(x, \alpha ; s, \tau, \mathfrak{a})}{\Delta(s)}+\widehat{\theta}(x ; s, \tau),
\end{aligned}
$$

where

$$
\begin{aligned}
& \psi(x, y ; s, \tau, \mathbf{L})=\langle\mathbf{L}, \boldsymbol{E}(y ; s, \tau)\rangle \cosh \sqrt{\frac{s}{\tau d}} x \\
& -\langle\mathbf{L}, \mathfrak{Q}(y ; s, \tau)\rangle \sinh \sqrt{\frac{s}{\tau d}} x, \\
& \widehat{\theta}(x ; s, \tau)=\frac{\psi(x, \alpha ; s, \tau, \mathfrak{a})}{\Delta(s)} \sqrt{\frac{\tau d}{s}} \int_{\alpha}^{\beta}\langle\mathfrak{b}, \mathfrak{c}(\beta-y ; s, \tau)\rangle F(y ; s, \tau) d y \\
& +\sqrt{\frac{\tau d}{s}} \int_{\alpha}^{x} \sinh \sqrt{\frac{s}{\tau d}}(x-y) F(y ; s, \tau) d y .
\end{aligned}
$$

For further computation, we rewrite $\widehat{\theta}(x ; s, \tau)$ as

$$
\begin{aligned}
\widehat{\theta}(x ; s, \tau)= & \sqrt{\frac{\tau d}{s}}\left(\int_{\alpha}^{x} \frac{\psi(x, \alpha ; s, \tau, \mathfrak{a})\langle\mathfrak{b}, \mathfrak{c}(\beta-y ; s, \tau)\rangle+\Delta(s) \sinh \sqrt{(s / \tau d)}(x-y)}{\Delta(s)} F(y ; s, \tau) d y\right) \\
& +\sqrt{\frac{\tau d}{s}} \int_{x}^{\beta} \frac{\psi(x, \alpha ; s, \tau, \mathfrak{a})\langle\mathfrak{b}, \mathfrak{c}(\beta-y ; s, \tau)\rangle}{\Delta(s)} F(y ; s, \tau) d y .
\end{aligned}
$$

Lemma 3. Let $\tau, s, x, y \in \mathbb{R}$ and $\mathbf{L} \in \mathbb{R}^{2}$. Then,

$$
\psi(x, y ; s, \tau, \mathbf{L})=\langle\mathbf{L}, \mathfrak{S}(y-x ; s, \tau)\rangle,
$$

$$
\begin{aligned}
& \Delta(s) \sinh \sqrt{\frac{s}{\tau d}}(x-y)=-\psi(x, \alpha ; s, \tau, \mathfrak{a})\langle\mathfrak{b}, \mathfrak{c}(\beta-y ; s, \tau)\rangle \\
& \quad+\langle\mathfrak{a}, \mathfrak{C}(\alpha-y ; s, \tau)\rangle \psi(x, \beta ; s, \tau, \mathfrak{b}) .
\end{aligned}
$$

Consequently, for each zero $s$ of the function $\Delta(s)$ sinh $\sqrt{(s / \tau d)}(x-y)$, one has

$$
\begin{aligned}
& \psi(x, \alpha ; s, \tau, \mathfrak{a})\langle\mathfrak{b}, \mathfrak{E}(\beta-y ; s, \tau)\rangle \\
& \quad=\langle\mathfrak{a}, \mathfrak{S}(\alpha-y ; s, \tau)\rangle \psi(x, \beta ; s, \tau, \mathfrak{b}),
\end{aligned}
$$

where $\psi$ is given by (57).
Proof. The first two conclusions of the lemma follow directly from the uniqueness theorem of the initial value problem for the second-order ordinary differential equations having constant coefficients.

For fixed $\tau, s, y \in \mathbb{R}$ and $L \in \mathbb{R}^{2}$, in view of (46) and (57), the functions $\langle\mathbf{L}, \boldsymbol{E}(y-x ; s, \tau)\rangle$ and $\psi(x, y ; s, \tau, \mathbf{L})$ are solutions to the following initial value problem:

$$
\begin{gathered}
\frac{d^{2} z(x)}{d x^{2}}-\frac{s}{\tau d} z(x)=0, \\
z(0)=\langle L, \mathfrak{S}(y ; s, \tau)\rangle, z^{\prime}(0)=-\sqrt{\frac{s}{\tau d}}\langle L, \mathfrak{L}(y ; s, \tau)\rangle .
\end{gathered}
$$

Thus, with the uniqueness of the solution to problem (63), we conclude (60).

It is easy to see that as functions in $x$, both sides of (61) are linear combinations of the functions $\cosh \sqrt{(s / \tau d)} x$, 
and $\sinh \sqrt{(s / \tau d)} x$ which are linearly independent solutions to the differential equation in (63). Thus, both sides of (61) solve this differential equation.

Moreover, in view of (57), both sides of (61) satisfy

$$
z(0)=-\Delta(s) \sinh \sqrt{\frac{s}{\tau d}} y, z^{\prime}(0)=\sqrt{\frac{s}{\tau d}} \cosh \sqrt{\frac{s}{\tau d}} y .
$$

Hence, by the uniqueness theorem, (61) holds true.

For each $s_{*}$ being a zero of the function $\Delta(s) \sinh$ $\sqrt{(s / \tau d)}(x-y)$, taking the limit in both sides of (61) as $s$ $\longrightarrow s_{*}$ gives (62).

Applying Lemma 3, (56) and (59) respectively can be reduced to

$$
\begin{aligned}
\widehat{\varphi}(x ; s, \tau)= & \frac{\mathbb{G}(s, \tau)\langle\mathfrak{b}, \mathfrak{E}(\beta-x ; s, \tau)\rangle}{\Delta(s)} \\
& -\frac{H(s, \tau)\langle\mathfrak{a}, \mathfrak{E}(\alpha-x ; s, \tau)\rangle}{\Delta(s)}+\widehat{\theta}(x ; s, \tau),
\end{aligned}
$$

$$
\begin{aligned}
\widehat{\theta}(x ; s, \tau)= & \sqrt{\frac{\tau d}{s}} \int_{\alpha}^{x} \frac{\langle\mathfrak{a}, \mathfrak{C}(\alpha-y ; s, \tau)\rangle\langle\mathfrak{b}, \mathfrak{C}(\beta-x ; s, \tau)\rangle}{\Delta(s)} F(y ; s, \tau) d y \\
& +\sqrt{\frac{\tau d}{s}} \int_{x}^{\beta} \frac{\langle\mathfrak{a}, \mathfrak{C}(\alpha-x ; s, \tau)\rangle\langle\mathfrak{b}, \mathfrak{C}(\beta-y ; s, \tau)\rangle}{\Delta(s)} F(y ; s, \tau) d y .
\end{aligned}
$$

Next, in order to obtain the solution to the initial value problem (34)-(37), we apply the inversion formula (33) to (65) and (66). In doing so, we suppose that there are nonzero simple roots $\left\{s_{k}\right\}_{k=1}^{\infty}$ of $\Delta(s)$. That is,

$$
\Delta\left(s_{k}\right)=0, \Delta^{\prime}\left(s_{k}\right) \neq 0, \quad k=1,2,3, \cdots
$$

Lemma 4. Suppose that (67) holds true. For each $x, y \in \mathbb{R}$ and $t, \tau>0$, we get

$$
\mathbb{N}^{-1}\left\{\frac{\langle\mathfrak{a}, \mathfrak{c}(x ; s, \tau)\rangle\langle\mathfrak{b}, \mathfrak{s}(y ; s, \tau)\rangle}{\sqrt{(s / \tau d)} \Delta(s)}\right\}=\Theta(x, y, t, \tau),
$$

where

$$
\Theta(x, y, t, \tau)=\Theta_{0}(x, y, \tau)+\sum_{k=1}^{\infty} \frac{e^{s_{k} t / \tau}\left\langle\mathfrak{a}, \mathfrak{E}\left(x ; s_{k}, \tau\right)\right\rangle\left\langle\mathfrak{b}, \mathfrak{\subseteq}\left(y ; s_{k}, \tau\right)\right\rangle}{\sqrt{s_{k} / \tau d} \Delta^{\prime}\left(s_{k}\right)},
$$

with

$$
\Theta_{0}(x, y)= \begin{cases}0 & \text { if } \frac{\langle\mathfrak{a}, \mathfrak{c}(x ; s, \tau)\rangle\langle\mathfrak{b}, \mathfrak{c}(y ; s, \tau)\rangle}{\sqrt{s / \tau d} \Delta(s)}=O(1), \\ \lim _{s \longrightarrow 0} \sqrt{s \tau d} \frac{\langle\mathfrak{a}, \mathfrak{S}(x ; s, \tau)\rangle\langle\mathfrak{b}, \mathfrak{c}(y ; s, \tau)\rangle}{\Delta(s)} & \text { if } \frac{\langle\mathfrak{a}, \mathfrak{s}(x ; s, \tau)\rangle\langle\mathfrak{b}, \mathfrak{c}(y ; s, \tau)\rangle}{\sqrt{s / \tau d} \Delta(s)}=O\left(\frac{1}{s}\right) .\end{cases}
$$

Proof. Let

$$
\mathfrak{R}(s, \tau)=\frac{\langle\mathfrak{a}, \mathfrak{C}(x ; s, \tau)\rangle\langle\mathfrak{b}, \mathfrak{c}(y ; s, \tau)\rangle}{\sqrt{s / \tau d} \Delta(s)} .
$$

Applying the inversion formula (33), we find

$$
\Theta(x, y, t)=\mathbb{N}^{-1}\{\mathfrak{R}(s, \tau)\}=\frac{1}{2 \pi i} \int_{c-i \infty}^{c+i \infty} e^{s t / \tau} \mathfrak{R}(s, \tau) d s, \quad c, \tau>0 .
$$

The last integral can be usually calculated by the residue theorem [54]. Hence,

$$
\Theta(x, y, t)=\sum_{\text {poles } s_{k} \text { of } \Re(s, \tau)} \operatorname{Res}\left[e^{s t / \tau} \mathfrak{R}(s, \tau) ; s_{k}\right]
$$

Recalling (67), each $s_{k}(k=1,2, \cdots)$ is a simple pole of $e^{s t / \tau} \Re(s, \tau)$. Therefore,

$\operatorname{Res}\left[e^{s t / \tau} \mathfrak{R}(s, \tau) ; s_{k}\right]=\frac{e^{s_{k} t / \tau}\left\langle\mathfrak{a}, \mathfrak{C}\left(x ; s_{k}, \tau\right)\right\rangle\left\langle\mathfrak{b}, \mathfrak{C}\left(y ; s_{k}, \tau\right)\right\rangle}{\sqrt{\left(s_{k} / \tau_{d}\right)} \Delta^{\prime}\left(s_{k}\right)}, \quad k=1,2, \cdots$

At $s=0$, we have

$$
\begin{aligned}
\operatorname{Res}\left[e^{s t / \tau} \mathfrak{R}(s, \tau) ; 0\right] & =\lim _{s \longrightarrow 0} s e^{s t / \tau} \mathfrak{R}(s, \tau) \\
& =\lim _{s \longrightarrow 0} \frac{s}{\Delta(s)} \frac{e^{s t / \tau}\langle\mathfrak{a}, \mathfrak{c}(x ; s, \tau)\rangle\langle\mathfrak{b}, \mathfrak{c}(y ; s, \tau)\rangle}{\sqrt{s / \tau d}} \\
& =\lim _{s \longrightarrow 0} \sqrt{s \tau d} \frac{\langle\mathfrak{a}, \mathfrak{\mathfrak { c }}(x ; s, \tau)\rangle\langle\mathfrak{b}, \mathfrak{c}(y ; s, \tau)\rangle}{\Delta(s)} .
\end{aligned}
$$


We see that either

$$
\begin{aligned}
\frac{\langle\mathfrak{a}, \mathfrak{S}(x ; s, \tau)\rangle\langle\mathfrak{b}, \mathfrak{s}(y ; s, \tau)\rangle}{\sqrt{(s / \tau d)} \Delta(s)} & =O(1) \text { or } \frac{\langle\mathfrak{a}, \mathfrak{c}(x ; s, \tau)\rangle\langle\mathfrak{b}, \mathfrak{c}(y ; s, \tau)\rangle}{\sqrt{(s / \tau d)} \Delta(s)} \\
& =O\left(\frac{1}{s}\right),
\end{aligned}
$$

as $s$ tends to 0 . Then, $s=0$ is either a removable singular point or a simple pole of $e^{s t / \tau} \mathfrak{R}(s, \tau)$.

Hence, substituting (74) and (75) in (73) gives the main conclusion of the Lemma, i.e., (69) and (70). $\square$

In view of (68) and (42), we have

$$
\mathbb{N}[\Theta(\alpha-y, \beta-x, t)]=\frac{\langle\mathfrak{a}, \mathfrak{c}(x-y, \beta)\rangle\langle\mathfrak{b}, \mathfrak{c}(\beta-x, s)\rangle}{\sqrt{(s / \tau d)} \Delta(s)},
$$

where $\Theta$ is defined by (69) and (70), and

$$
F(y ; s, \tau)=\frac{1}{d} \mathbb{N}\left[\frac{\lambda(y, t)}{\left(t^{m} / \tau^{m}+\tau^{m}\right)^{\rho}}\right](y ; s, \tau)-\frac{1}{\tau d} \eta(y) .
$$

Hence, (66) can be rewritten as

$$
\begin{aligned}
\widehat{\theta}(x ; s, \tau)= & -\frac{1}{d} \int_{\alpha}^{x} \mathbb{N}[\Theta(\alpha-y, \beta-x, t)] \mathbb{N}\left[\frac{r(y, t)}{\left(t^{m} / \tau^{m}+\tau^{m}\right)^{\rho}}\right] d y \\
& -\frac{1}{\tau d} \int_{\alpha}^{x} \mathbb{N}[\Theta(\alpha-y, \beta-x, t)] \eta(y) d y \\
& -\frac{1}{d} \int_{x}^{\beta} \mathbb{N}[\Theta(\alpha-x, \beta-y, t)] \mathbb{N}\left[\frac{r(y, t)}{\left(t^{m} / \tau^{m}+\tau^{m}\right)^{\rho}}\right] d y \\
& -\frac{1}{\tau d} \int_{x}^{\beta} \mathbb{N}[\Theta(\alpha-x, \beta-y, t)] \eta(y) d y .
\end{aligned}
$$

By the convolution formula (31), the inverse natural transform of (79) is

$$
\begin{aligned}
\theta(x, t, \tau)= & -\frac{1}{\tau d} \int_{\alpha}^{x} \int_{0}^{t} \Theta(\alpha-y, \beta-x, t-\zeta) \frac{r(y, \zeta)}{\left(\zeta^{m} / \tau^{m}+\tau^{m}\right)^{\rho}} d \zeta d y \\
& -\frac{1}{\tau d} \int_{\alpha}^{x} \Theta(\alpha-y, \beta-x, t) \eta(y) d y \\
& -\frac{1}{\tau d} \int_{x}^{\beta} \int_{0}^{t} \Theta(\alpha-x, \beta-y, t-\zeta) \frac{r(y, \zeta)}{\left(\zeta^{m} / \tau^{m}+\tau^{m}\right)^{\rho}} d \zeta d y \\
& -\frac{1}{\tau d} \int_{x}^{\beta} \Theta(\alpha-x, \beta-y, t) \eta(y) d y .
\end{aligned}
$$

That is,

$$
\begin{aligned}
\theta(x, t, \tau)= & -\frac{1}{\tau d} \int_{\alpha}^{x} \Theta_{0}(\alpha-y, \beta-x) \eta(y) d y-\frac{1}{\tau d} \int_{x}^{\beta} \Theta_{0}(\alpha-x, \beta-y) \eta(y) d y \\
& -\frac{1}{\tau d} \int_{\alpha}^{x} \int_{0}^{t} \Theta_{0}(\alpha-y, \beta-x) \frac{r(y, \zeta)}{\left(\zeta^{m} / \tau^{m}+\tau^{m}\right)^{\rho}} d \zeta d y \\
& -\frac{1}{\tau d} \int_{x}^{\beta} \int_{0}^{t} \Theta_{0}(\alpha-x, \beta-y) \frac{r(y, \zeta)}{\left(\zeta^{m} / \tau^{m}+\tau^{m}\right)^{\rho}} d \zeta d y \\
& -\frac{1}{\tau d} \sum_{k=1}^{\infty} \frac{\left\langle\mathfrak{b}, \mathfrak{C}\left(\beta-x ; s_{k}, \tau\right)\right\rangle}{\sqrt{s_{k} / \tau d} \Delta^{\prime}\left(s_{k}\right)} \\
& \cdot \int_{\alpha}^{x} \int_{0}^{t} \frac{e^{s_{k}(t-\zeta) / \tau} r(y, \zeta)\left\langle\mathfrak{a}, \mathfrak{S}\left(\alpha-y ; s_{k}, \tau\right)\right\rangle}{\left(\zeta^{m} / \tau^{m}+\tau^{m}\right)^{\rho}} d \zeta d y \\
& -\frac{1}{\tau d} \sum_{k=1}^{\infty} \frac{\left\langle\mathfrak{a}, \mathfrak{C}\left(\alpha-x ; s_{k}, \tau\right)\right\rangle}{\sqrt{s_{k} / \tau d} \Delta^{\prime}\left(s_{k}\right)} \\
& \cdot \int_{x}^{\beta} \int_{0}^{t} \frac{e^{s_{k}(t-\zeta) / \tau} r(y, \zeta)\left\langle\mathfrak{b}, \mathfrak{c}\left(\beta-y ; s_{k}, \tau\right)\right\rangle}{\left(\zeta^{m} / \tau^{m}+\tau^{m}\right)^{\rho}} d \zeta d y \\
& -\frac{1}{\tau d} \sum_{k=1}^{\infty} \frac{e^{s_{k} t / \tau}\left\langle\mathfrak{b}, \mathfrak{C}\left(\beta-x ; s_{k}, \tau\right)\right\rangle}{\sqrt{s_{k} / \tau d} \Delta^{\prime}\left(s_{k}\right)} \int_{\alpha}^{x}\left\langle\mathfrak{a}, \mathfrak{C}\left(\alpha-y ; s_{k}, \tau\right)\right\rangle \eta(y) d y \\
& -\frac{1}{\tau d} \sum_{k=1}^{\infty} \frac{e^{s_{k} t / \tau}\left\langle\mathfrak{a}, \mathfrak{C}\left(\alpha-x ; s_{k}, \tau\right)\right\rangle}{\sqrt{s_{k} / \tau d} \Delta^{\prime}\left(s_{k}\right)} \int_{x}^{\beta}\left\langle\mathfrak{b}, \mathfrak{C}\left(\beta-y ; s_{k}, \tau\right)\right\rangle \eta(y) d y .
\end{aligned}
$$

From Lemma 3, one has

$$
\begin{aligned}
& \langle\mathfrak{a}, \mathfrak{C}(\alpha-x ; s, \tau)\rangle\langle\mathfrak{b}, \mathfrak{S}(\beta-y ; s, \tau)\rangle \\
& \quad=\langle\mathfrak{a}, \mathfrak{C}(\alpha-y ; s, \tau)\rangle\langle\mathfrak{b}, \mathfrak{S}(\beta-x ; s, \tau)\rangle,
\end{aligned}
$$

at $s=0$ and $s=s_{k}(k=1,2, \cdots)$ the zeros of $\Delta(s) \sinh \sqrt{s / \tau d}($ $x-y)$. That results in

$$
\begin{gathered}
\Theta_{0}(\alpha-y, \beta-x)=\Theta_{0}(\alpha-x, \beta-y), \\
\left\langle\mathfrak{a}, \mathfrak{S}\left(\alpha-x ; s_{k}, \tau\right)\right\rangle \int_{x}^{\beta}\left\langle\mathfrak{b}, \mathfrak{S}\left(\beta-y ; s_{k}, \tau\right)\right\rangle \vartheta(y) d y \\
=\left\langle\mathfrak{b}, \mathfrak{S}\left(\beta-x ; s_{k}, \tau\right)\right\rangle \int_{x}^{\beta}\left\langle\mathfrak{a}, \mathfrak{\mathfrak { C }}\left(\alpha-y ; s_{k}, \tau\right)\right\rangle \vartheta(y) d y .
\end{gathered}
$$

The first conclusion is obvious when $\Theta_{0}=0$ in (70). Thus, (81) can be simplified as

$$
\begin{aligned}
\theta(x, t, \tau)= & -\frac{1}{\tau d} \int_{\alpha}^{\beta} \Theta_{0}(\alpha-x, \beta-y) \eta(y) d y \\
& -\frac{1}{\tau d} \int_{\alpha}^{\beta} \int_{0}^{t} \Theta_{0}(\alpha-x, \beta-y) \frac{r(y, \zeta)}{\left(\zeta^{m} / \tau^{m}+\tau^{m}\right)^{\rho}} d \zeta d y \\
& -\frac{1}{\tau d} \sum_{k=1}^{\infty} \frac{\left\langle\mathfrak{b}, \mathfrak{C}\left(\beta-x ; s_{k}, \tau\right)\right\rangle}{\sqrt{s_{k} / \tau d} \Delta^{\prime}\left(s_{k}\right)} \\
& \cdot \int_{\alpha}^{\beta} \int_{0}^{t} \frac{e^{s_{k}(t-\zeta) / \tau} r(y, \zeta)\left\langle\mathfrak{a}, \mathfrak{C}\left(\alpha-y ; s_{k}, \tau\right)\right\rangle}{\left(\zeta^{m} / \tau^{m}+\tau^{m}\right)^{\rho}} d \zeta d y \\
& -\frac{1}{\tau d} \sum_{k=1}^{\infty} \frac{e^{s_{k} t / \tau}\left\langle\mathfrak{b}, \mathfrak{C}\left(\beta-x ; s_{k}, \tau\right)\right\rangle}{\sqrt{s_{k} / \tau d} \Delta^{\prime}\left(s_{k}\right)} \int_{\alpha}^{\beta}\left\langle\mathfrak{a}, \mathfrak{C}\left(\alpha-y ; s_{k}, \tau\right)\right\rangle \eta(y) d y .
\end{aligned}
$$


Next, we return to (65). Using (26) (for $n=1$ ) and (51), (65) can be rewritten as

$$
\begin{aligned}
\widehat{\varphi}(x ; s, \tau)= & s \mathbb{N}[\lambda(t, \tau) \zeta(t)] \frac{\langle\mathfrak{b}, \mathfrak{C}(\beta-x ; s, \tau)\rangle}{s \Delta(s)} \\
& -s \mathbb{N}[\lambda(t, \tau) \xi(t)] \frac{\langle\mathfrak{a}, \mathfrak{C}(\alpha-x ; s, \tau)\rangle}{s \Delta(s)}+\widehat{\theta}(x ; s, \tau) \\
= & \left(\tau \mathbb{N}\left[\frac{d}{d t}(\lambda(t, \tau) \zeta(t))\right]+\lambda(0, \tau) \zeta(0)\right) \frac{\langle\mathfrak{b}, \mathfrak{C}(\beta-x ; s, \tau)\rangle}{s \Delta(s)} \\
& -\left(\tau \mathbb{N}\left[\frac{d}{d t}(\lambda(t, \tau) \xi(t))\right]+\lambda(0, \tau) \xi(0)\right) \\
& \cdot \frac{\langle\mathfrak{a}, \mathfrak{C}(\alpha-x ; s, \tau)\rangle}{s \Delta(s)}+\widehat{\theta}(x ; s, \tau),
\end{aligned}
$$

where $\widehat{\theta}(x ; s, \tau)$ is given in (66). Now, we can obtain the solution $\varphi(x, t)$ of Problem (34)-(37) by operating the inversion formula (33) in (85). In doing so, we need the following lemma.

Lemma 5. Assume that (67) holds true. Then, for each $y \in$ $\mathbb{R}, t, \tau>0$ and $\mathbf{L} \in \mathbb{R}^{2}$, we get

$$
\mathbb{N}^{-1}\left\{\frac{\langle\mathbf{L}, \mathfrak{c}(y ; s, \tau)\rangle}{s \Delta(s)}\right\}=\Phi(y, t ; \mathbf{L})
$$

where

$$
\begin{gathered}
\Phi(y, t ; \mathbf{L})=\Phi_{0}(y ; \mathbf{L})+\sum_{k=1}^{\infty} \frac{e^{s_{k} t / \tau}\left\langle\mathbf{L}, \mathfrak{E}\left(y ; s_{k}, \tau\right)\right\rangle}{s_{k} \Delta^{\prime}\left(s_{k}\right)}, \\
\Phi_{0}(y ; \mathbf{L})=\left\{\begin{array}{cc}
\lim _{s \longrightarrow 0} \frac{\langle\mathbf{L}, \mathfrak{c}(y ; s, \tau)\rangle}{\Delta(s)}, & \text { if } \frac{\langle\mathbf{L}, \mathfrak{c}(y ; s, \tau)\rangle}{s \Delta(s)}=O\left(\frac{1}{s}\right) \\
\lim _{s \longrightarrow 0} \frac{\partial}{\partial s}\left(\frac{s\langle\mathbf{L}, \mathfrak{c}(y ; s, \tau)\rangle}{\Delta(s)}\right), & \text { if } \frac{\langle\mathbf{L}, \mathfrak{c}(y ; s, \tau)\rangle}{s \Delta(s)}=O\left(\frac{1}{s^{2}}\right)
\end{array}\right\} .
\end{gathered}
$$

Proof. The proof is similar to Lemma 4.

$$
\text { From Lemma 5, we see }
$$
that-

$\mathbb{N}^{-1}\{\langle\mathfrak{b}, \mathfrak{C}(\beta-x ; s, \tau)\rangle / s \Delta(s)\}=\Phi(\beta-x, t ; \mathfrak{b}), \mathbb{N}^{-1}\{\langle\mathfrak{a}, \mathfrak{C}(\alpha$ $-x ; s, \tau)\rangle / s \Delta(s)\}=\Phi(\alpha-x, t ; \mathfrak{a})$.

Hence, in view of the convolution formula (31) and the inversion of natural transform (33), inverting (85) yields

$$
\begin{aligned}
\varphi(x, t, \tau)= & \int_{0}^{t} \Phi(\beta-x, t-\varsigma ; \mathfrak{b})\left(\tilde{\zeta}^{\prime}(\varsigma)+\tilde{\zeta}(0) \delta_{0}(\varsigma)\right) d \varsigma \\
& -\int_{0}^{t} \Phi(\alpha-x, t-\varsigma ; \mathfrak{a})\left(\tilde{\xi}^{\prime}(\varsigma)+\tilde{\xi}(0) \delta_{0}(\varsigma)\right) d \varsigma \\
& +\theta(x, t, \tau),
\end{aligned}
$$

where $\tilde{\zeta}=\lambda(t, \tau) \zeta(t), \tilde{\xi}=\lambda(t, \tau) \xi(t), \delta_{0}$ is the well-known Dirac delta function, and $\theta(x, t, \tau)$ is given by (84). Then, using the basic property of the Dirac delta function, that is, $\delta_{0}(\varsigma) \Phi(\varsigma)=\Phi(0)$, results in

$$
\begin{aligned}
\varphi(x, t, \tau)= & \int_{0}^{t} \Phi(\beta-x, t-\varsigma ; \mathfrak{b}) \widetilde{\zeta^{\prime}}(\varsigma) d \varsigma+\tilde{\zeta}(0) \Phi(\beta-x, t ; \mathfrak{b}) \\
& -\int_{0}^{t} \Phi(\alpha-x, t-\varsigma ; \mathfrak{a}) \widetilde{\xi}^{\prime}(\varsigma) d \varsigma-\tilde{\xi}(0) \Phi(\alpha-x, t ; \mathfrak{a}) \\
& +\theta(x, t, \tau)
\end{aligned}
$$

Integrating by parts gives

$$
\begin{aligned}
\varphi(x, t, \tau)= & \lambda(t, \tau) \zeta(t) \Phi(\beta-x, 0 ; \mathfrak{b}) \\
& -\int_{0}^{t} \lambda(\varsigma, \tau) D_{2} \Phi(\beta-x, t-\varsigma ; \mathfrak{b}) \zeta(\varsigma) d \varsigma \\
& -\lambda(t, \tau) \Phi(\alpha-x, 0 ; \mathfrak{a}) \xi(t) \\
& +\int_{0}^{t} \lambda(\varsigma, \tau) D_{2} \Phi(\alpha-x, t-\varsigma ; \mathfrak{a}) \xi(\varsigma) d \varsigma+\theta(x, t, \tau),
\end{aligned}
$$

where $D_{2}=(\partial / \partial t) \Phi(x, t ; b)$. Substituting from (87) gives

$$
\begin{aligned}
\varphi(x, t, \tau)= & \lambda(t, \tau) \zeta(t)\left(\Phi_{0}(\beta-x ; \mathfrak{b})+\sum_{k=1}^{\infty} \frac{\left\langle\mathfrak{b}, \mathfrak{c}\left(\beta-x ; s_{k}, \tau\right)\right\rangle}{s_{k} \Delta^{\prime}\left(s_{k}\right)}\right) \\
& -\int_{0}^{t} \lambda(\varsigma, \tau) \zeta(\varsigma)\left(\sum_{k=1}^{\infty} \frac{e^{s_{k}(t-\varsigma) / \tau}\left\langle\mathfrak{b}, \mathfrak{c}\left(\beta-x ; s_{k}, \tau\right)\right\rangle}{\Delta^{\prime}\left(s_{k}\right)}\right) d \varsigma \\
& -\lambda(t, \tau) \xi(t)\left(\Phi_{0}(\alpha-x ; \mathfrak{a})+\sum_{k=1}^{\infty} \frac{\left\langle\mathfrak{a}, \mathfrak{c}\left(\alpha-x ; s_{k}, \tau\right)\right\rangle}{s_{k} \Delta^{\prime}\left(s_{k}\right)}\right) \\
& +\int_{0}^{t} \lambda(\varsigma, \tau) \xi(\varsigma)\left(\sum_{k=1}^{\infty} \frac{e^{s_{k}(t-\varsigma) / \tau}\left\langle\mathfrak{a}, \mathfrak{\complement}\left(\alpha-x ; s_{k}, \tau\right)\right\rangle}{\Delta^{\prime}\left(s_{k}\right)}\right) d \varsigma \\
& +\theta(x, t, \tau),
\end{aligned}
$$

with $\theta(x, t, \tau)$ is given by (84). This result can be rewritten as

$$
\begin{aligned}
\varphi(x, t, \tau)= & \lambda(t, \tau) \zeta(t) \Phi_{0}(\beta-x ; \mathfrak{b}) \\
& +\sum_{k=1}^{\infty} \frac{\Gamma_{k} \zeta(t)}{s_{k} \Delta^{\prime}\left(s_{k}\right)}\left\langle\mathfrak{b}, \mathfrak{c}\left(\beta-x ; s_{k}, \tau\right)\right\rangle \\
& -\lambda(t, \tau) \xi(t) \Phi_{0}(\alpha-x ; \mathfrak{a}) \\
& -\sum_{k=1}^{\infty} \frac{\Gamma_{k} \xi(t)}{s_{k} \Delta^{\prime}\left(s_{k}\right)}\left\langle\mathfrak{a}, \mathfrak{s}\left(\alpha-x ; s_{k}, \tau\right)\right\rangle+\theta(x, t, \tau)
\end{aligned}
$$


where $\Gamma_{k}$ is the operator defined as

$$
\Gamma_{k} \phi(t)=\lambda(t, \tau) \phi(t)-s_{k} \int_{0}^{t} \lambda(\varsigma, \tau) e^{s_{k}(t-\varsigma) / \tau} \phi(\varsigma) d \varsigma .
$$

The integral in (94) is the Laplacian convolution formula for $\lambda(t, \tau) \phi(t)$ with $e^{s_{k} t / \tau}$. As a result, (93), together with (84) and (94), expresses the solution of Problem (34)-(37).

Remark 6. When $\rho=0$ and $r_{j}=v_{j}=0$, for all $j=1, \cdots, n$, Problem (34)-(37) and its solution

$$
\begin{aligned}
\varphi(x, t, \tau=1)= & \zeta(t) \Phi_{0}(\beta-x ; \mathfrak{b})+\sum_{k=1}^{\infty} \frac{\widetilde{\Gamma}_{k} \zeta(t)}{s_{k} \Delta^{\prime}\left(s_{k}\right)}\left\langle\mathfrak{b}, \mathfrak{C}\left(\beta-x, s_{k}\right)\right\rangle \\
& -\xi(t) \Phi_{0}(\alpha-x ; \mathfrak{a})-\sum_{k=1}^{\infty} \frac{\widetilde{\Gamma}_{k} \xi(t)}{s_{k} \Delta^{\prime}\left(s_{k}\right)}\left\langle\mathfrak{a}, \mathfrak{C}\left(\alpha-x, s_{k}\right)\right\rangle \\
& +\theta(x, t),
\end{aligned}
$$

with $\Phi_{0}(\alpha-x ; \mathfrak{a}), \mathfrak{c}\left(y, s_{k}\right)=\mathfrak{C}\left(y ; s_{k}, \tau=1\right)$ defined as (88), (46), respectively,

$$
\begin{aligned}
\widetilde{\Gamma}_{k} \varphi(t)= & \varphi(t)-s_{k} \int_{0}^{t} e^{s_{k}(t-\varsigma)} \varphi(\varsigma) d \varsigma, \\
\theta(x, t)= & -\frac{1}{d} \int_{\alpha}^{\beta} \Theta_{0}(\alpha-x, \beta-y) \eta(y) d y \\
& -\sum_{k=1}^{\infty} \frac{e^{s_{k} t}\left\langle\mathfrak{b}, \mathfrak{C}\left(\beta-x ; s_{k}, 1\right)\right\rangle}{\sqrt{d s_{k}} \Delta^{\prime}\left(s_{k}\right)} \int_{\alpha}^{\beta}\left\langle\mathfrak{a}, \mathfrak{C}\left(\alpha-y ; s_{k}, 1\right)\right\rangle \eta(y) d y,
\end{aligned}
$$

are reduced to that in Section 3 of [38].

2.1. Illustrative Examples. Here, we discuss two illustrative test cases to show the accuracy and effectiveness of our technique.

Example 1. Heat equation with zero temperatures at finite ends.

The following initial boundary value problem with homogeneous Dirichlet boundary conditions

$$
\begin{gathered}
\frac{\partial \varphi}{\partial t}=d \frac{\partial^{2} \varphi}{\partial x^{2}}, \quad x \in(0, L), t>0, \\
\varphi(x, 0)=f(x), \quad x \in[0, L], \\
\varphi(0, t)=0, \varphi(L, t)=0, \quad t \geq 0,
\end{gathered}
$$

is a special case of the one-layer diffusion system (34)-(37) when $\alpha=0, \beta=L, \imath=1, \iota=0, \ell=1, l=0$ and $r(x, t)=\zeta(t)=$ $\xi(t)=0, \eta(x)=f(x)$. For simplicity, we will take $\tau=1$. Thus, from (93), we have $\varphi(x, t)=\theta(x, t)$. From (46), we have $\mathfrak{a}$ $=\mathfrak{b}=(1,0)$,

$$
\begin{aligned}
\mathfrak{L}(x ; s) & =\left(\cosh \sqrt{\frac{s}{d}} x, \sqrt{\frac{s}{d}} \sinh \sqrt{\frac{s}{d}} x\right), \quad \mathfrak{c}(x ; s) \\
& =\left(\sinh \sqrt{\frac{s}{d}} x, \sqrt{\frac{s}{d}} \cosh \sqrt{\frac{s}{d}} x\right) .
\end{aligned}
$$

Hence, (54) yields

$$
\Delta(s)=\sinh \sqrt{\frac{s}{d}} L, \quad \Delta^{\prime}(s)=\frac{L}{2 d} \sqrt{\frac{d}{s}} \cosh \sqrt{\frac{s}{d}} L .
$$

So, we have

$$
\Delta\left(s_{k}\right)=0, \Delta^{\prime}\left(s_{k}\right) \neq 0, \quad k=1,2,3, \cdots,
$$

at

$$
s_{k}=-\frac{k^{2} \pi^{2} d}{L^{2}}, \quad k=1,2,3, \cdots
$$

Moreover,

$\frac{\langle\mathfrak{a}, \mathfrak{c}(x ; s)\rangle\langle\mathfrak{b}, \mathfrak{c}(y ; s)\rangle}{\sqrt{s / d} \Delta(s)}=\frac{\sinh \sqrt{s / d} x \sinh \sqrt{s / d} y}{\sqrt{s / d} \sinh \sqrt{s / d} L}=O(1)$,

as $s \longrightarrow 0$. Therefore, $(70)$ gives $\Theta_{0}(x, y)=0$. Further,

$$
\begin{aligned}
\left\langle\mathfrak{a}, \mathfrak{C}\left(-y ; s_{k}\right)\right\rangle & =-i \sin \frac{k \pi}{L} y,\left\langle\mathfrak{b}, \mathfrak{C}\left(L-x ; s_{k}\right)\right\rangle \\
& =(-1)^{k+1} i \sin \frac{k \pi}{L} x, \quad k=1,2,3, \cdots \\
\sqrt{\frac{s_{k}}{d}} \Delta^{\prime}\left(s_{k}\right) & =(-1)^{k} \frac{L}{2 d}, \quad k=1,2,3, \cdots
\end{aligned}
$$

Finally, from (80), we get

$\varphi(x, t)=\theta(x, t)=-1 / d \sum_{k=1}^{\infty} e^{s_{k} t}\left\langle\mathfrak{b}, \mathfrak{C}\left(L-x ; s_{k}\right)\right\rangle / \sqrt{s_{k} / d} \Delta$ $'\left(s_{k}\right) \int_{0}^{L}\left\langle\mathfrak{a}, \mathfrak{c}\left(-y ; s_{k}\right)\right\rangle f(y) d y=\sum_{k=0}^{\infty}\left[2 / L \int_{0}^{L} f(y) \sin (k \pi y / L) d y\right.$ ]$e^{-\left(k^{2} \pi^{2} d / L^{2}\right) t} \sin (k \pi / L) x$,

which recovers the solution to problem (97) obtained via the separation of variables method in [55]. 
Example 2. Heat flow with sources and homogeneous boundary conditions.

The following initial boundary value problem

$$
\begin{gathered}
\frac{\partial \varphi}{\partial t}=d \frac{\partial^{2} \varphi}{\partial x^{2}}+\left(t^{m}+1\right)^{-\rho} e^{-t} \sin 3 x, x \in(0, \pi), \quad t>0 \\
\varphi(x, 0)=f(x), x \in[0, \pi] \\
\varphi(0, t)=0 . \quad \varphi(\pi, t)=0, \quad t \geq 0
\end{gathered}
$$

is a special case of the one-layer diffusion system (34)-(37) when $\alpha=0, \beta=\pi, d=\tau=1, \imath=1, \iota=0, \ell=1, l=0$ and $r(x, t)=e^{-t} \sin 3 x, \eta(x)=f(x), \zeta(t)=\xi(t)=0$. Thus, from (93), we have $\varphi(x, t)=\theta(x, t)$. From (46), we have $\mathfrak{a}=\mathfrak{b}=(1,0)$,

$$
\begin{aligned}
\mathfrak{L}(x ; s) & =(\cosh \sqrt{s} x, \sqrt{s} \sinh \sqrt{s} x), \mathfrak{c}(x ; s) \\
& =(\sinh \sqrt{s} x, \sqrt{s} \cosh \sqrt{s} x) .
\end{aligned}
$$

Hence, (54) yields

$$
\Delta(s)=\sinh \sqrt{s} \pi, \Delta^{\prime}(s)=\frac{\pi}{2} \sqrt{\frac{1}{s}} \cosh \sqrt{s} \pi .
$$

So, $\Delta(s)$ has simple zeros at $s_{k}=-k^{2}, k=1,2,3, \cdots$. Further,

$$
\begin{aligned}
\left\langle\mathfrak{a}, \mathfrak{C}\left(-y ; s_{k}\right)\right\rangle & =-i \sin k y,\left\langle\mathfrak{b}, \mathfrak{c}\left(\pi-x ; s_{k}\right)\right\rangle \\
& =(-1)^{k+1} i \sin k x, \quad k=1,2,3, \cdots, \\
\sqrt{s_{k}} \Delta^{\prime}\left(s_{k}\right) & =(-1)^{k} \frac{\pi}{2}, \quad k=1,2,3, \cdots .
\end{aligned}
$$

On the other hand,

$$
\frac{\langle\mathfrak{a}, \mathfrak{c}(x ; s)\rangle\langle\mathfrak{b}, \mathfrak{c}(y ; s)\rangle}{\sqrt{s} \Delta(s)}=\frac{\sinh \sqrt{s} x \sinh \sqrt{s} y}{\sqrt{s} \sinh \sqrt{s} \pi}=O(1)
$$

as $s \longrightarrow 0$. Therefore, $(70)$ gives $\Theta_{0}(x, y)=0$.
Finally, from (80), we get

$$
\begin{aligned}
\varphi(x, t)= & \theta(x, t)=\sum_{k=1}^{\infty}\left[\frac{2}{\pi} \int_{0}^{\pi} \int_{0}^{t} \frac{e^{\left(k^{2}-1\right) z} \sin 3 y \sin k y}{\left(z^{m}+1\right)^{\rho}} d z d y\right] e^{-k^{2} t} \sin k x \\
& +\sum_{k=0}^{\infty}\left[\frac{2}{\pi} \int_{0}^{\pi} f(y) \sin k y d y\right] e^{-k^{2} t} \sin k x \\
= & {\left[\int_{0}^{t} \frac{e^{8 z}}{\left(z^{m}+1\right)^{\rho}}\right] e^{-9 t} \sin 3 x } \\
& +\sum_{k=0}^{\infty}\left[\frac{2}{\pi} \int_{0}^{\pi} f(y) \sin k y d y\right] e^{-k^{2} t} \sin k x \\
= & \sum_{k=o}^{\infty} a_{k}(t) \sin k x,
\end{aligned}
$$

where

$$
a_{k}(t)= \begin{cases}a_{k}(0) e^{-k^{2} t}, & k \neq 3, \\ {\left[a_{3}(0)+a(t)\right] e^{-9 t},} & k=3,\end{cases}
$$

with

$$
a_{k}(0)=\frac{2}{\pi} \int_{0}^{t} f(y) \sin k y d y, \quad a(t)=\frac{2}{\pi} \int_{0}^{t} \frac{e^{8 z}}{\left(z^{m}+1\right)^{\rho}} d z .
$$

When $\rho=0$, formula (109) recovers the solution to problem (104) (when $\rho=0$ ) obtained via the eigenfunction expansion method in [55].

\section{Multilayer Nonhomogeneous Diffusion System}

Here, we are seeking the solution of our main problem defined in (2)-(8), which was converted into a sequence of initial boundary value problems (9)-(11). For the convenience of the reader and in order to draw the full picture in an easy way, we start with solving the bilayer diffusion problem in the following subsection; then, we move to the general case in Section 3.2.

3.1. Solution of a Two-Layer Problem. For the two-layer problem, we have

$$
\begin{gathered}
\frac{\partial \varphi_{1}}{\partial t}=d_{1} \frac{\partial^{2} \varphi_{1}}{\partial x^{2}}+\lambda(t, \tau) r_{1}(x, t), \quad x \in\left(x_{0}, x_{1}\right), t, \tau>0 \\
\varphi_{1}(x, 0)=\eta_{1}(x), \quad x \in\left[x_{0}, x_{1}\right] \\
\imath \varphi_{1}\left(x_{0}, t\right)+\iota \frac{\partial \varphi_{1}}{\partial x}\left(x_{0}, t\right)=\lambda(t, \tau) \zeta_{1}(t), \quad t \geq 0, \tau>0
\end{gathered}
$$


$v_{1} \varphi_{1}\left(x_{1}, t\right)+\mu_{1} \frac{\partial \varphi_{1}}{\partial x}\left(x_{1}, t\right)=\lambda(t, \tau) \xi_{1}(t), \quad t \geq 0, \tau>0$

$$
\frac{\partial \varphi_{2}}{\partial t}=d_{2} \frac{\partial^{2} \varphi_{2}}{\partial x^{2}}+\lambda(t, \tau) r_{2}(x, t), \quad x \in\left(x_{1}, x_{2}\right), t, \tau>0,
$$

$$
\varphi_{2}(x, 0)=\eta_{2}(x), \quad x \in\left[x_{1}, x_{2}\right]
$$

$v_{2} \varphi_{2}\left(x_{1}, t\right)+\mu_{2} \frac{\partial \varphi_{2}}{\partial x}\left(x_{1}, t\right)=\lambda(t, \tau) \zeta_{2}(t), \quad t \geq 0, \tau>0$

$$
\ell \varphi_{2}\left(x_{2}, t\right)+l \frac{\partial \varphi_{2}}{\partial x}\left(x_{2}, t\right)=\lambda(t, \tau) \xi_{2}(t), \quad t \geq 0, \tau>0 .
$$

Similar to what we denote in Section 2, we define the following vector notation $\mathfrak{a}_{1}=(\iota, \iota), \mathfrak{b}_{1}=\left(v_{1}, \mu_{1}\right), \mathfrak{a}_{2}=\left(\nu_{2}, \mu_{2}\right)$, $\mathfrak{b}_{2}=(\ell, l)$, and

$$
\begin{aligned}
& \mathfrak{Q}_{1}(y ; s, \tau)=\left(\cosh \sqrt{\frac{s}{\tau d_{1}}} y, \sqrt{\frac{s}{\tau d_{1}}} \sinh \sqrt{\frac{s}{\tau d_{1}} y}\right), \\
& \mathfrak{Q}_{2}(y ; s, \tau)=\left(\cosh \sqrt{\frac{s}{\tau d_{2}}} y, \sqrt{\frac{s}{\tau d_{2}}} \sinh \sqrt{\frac{s}{\tau d_{2}} y}\right) \text {, } \\
& \mathfrak{\mho}_{1}(y ; s, \tau)=\left(\sinh \sqrt{\frac{s}{\tau d_{1}}} y, \sqrt{\frac{s}{\tau d_{1}}} \cosh \sqrt{\frac{s}{\tau d_{1}} y}\right) \text {, } \\
& \mathfrak{c}_{2}(y ; s, \tau)=\left(\sinh \sqrt{\frac{s}{\tau d_{2}}} y, \sqrt{\frac{s}{\tau d_{2}}} \cosh \sqrt{\frac{s}{\tau d_{2}} y}\right) \text {. }
\end{aligned}
$$

Also, analogues to (54), define

$$
\begin{aligned}
\Delta_{1}(s)= & \left\langle\mathfrak{b}_{1}, \mathfrak{b}_{1}\left(x_{1}, s, \tau\right)\right\rangle\left\langle\mathfrak{a}_{1}, \mathfrak{Q}_{1}\left(x_{0}, s, \tau\right)\right\rangle \\
& -\left\langle\mathfrak{a}_{1}, \mathfrak{b}_{1}\left(x_{0}, s, \tau\right)\right\rangle\left\langle\mathfrak{b}_{1}, \mathfrak{Q}_{1}\left(x_{1}, s, \tau\right)\right\rangle, \\
\Delta_{2}(s)= & \left\langle\mathfrak{b}_{2}, \mathfrak{b}_{2}\left(x_{2}, s, \tau\right)\right\rangle\left\langle\mathfrak{a}_{2}, \mathfrak{\Omega}_{2}\left(x_{1}, s, \tau\right)\right\rangle \\
& -\left\langle\mathfrak{a}_{2}, \mathfrak{b}_{2}\left(x_{1}, s, \tau\right)\right\rangle\left\langle\mathfrak{b}_{2}, \mathfrak{Q}_{2}\left(x_{2}, s, \tau\right)\right\rangle .
\end{aligned}
$$

Further, similar to (67), suppose that there are nonzero simple roots $\left\{s_{k}^{(1)}\right\}_{k=1}^{\infty}$ and $\left\{s_{k}^{(2)}\right\}_{k=1}^{\infty}$ of the functions $\Delta_{1}(s)$ and $\Delta_{2}(s)$, respectively. That is,

$$
\begin{aligned}
\Delta_{1}\left(s_{k}^{(1)}\right) & =0, \Delta_{1}{ }^{\prime}\left(s_{k}^{(1)}\right) \neq 0, \Delta_{2}\left(s_{k}^{(2)}\right) \\
& =0, \Delta_{2}{ }^{\prime}\left(s_{k}^{(2)}\right) \neq 0 \quad(k=1,2, \cdots) .
\end{aligned}
$$

Therefore, according to (84), we obtain

$$
\begin{aligned}
& \theta_{1}(x, t, \tau)=-\frac{1}{\tau d_{1}} \int_{x_{0}}^{x_{1}} \Theta_{0}^{(1)}\left(x_{0}-x, x_{1}-y\right) \eta_{1}(y) d y \\
& -\frac{1}{\tau d_{1}} \int_{x_{0}}^{x_{1}} \int_{0}^{t} \Theta_{0}^{(1)}\left(x_{0}-x, x_{1}-y\right) \frac{r_{1}(y, \varsigma)}{\left(\varsigma^{m} / \tau^{m}+\tau^{m}\right)^{\rho}} d \varsigma d y \\
& -\frac{1}{\tau d_{1}} \sum_{k=1}^{\infty} \frac{\left\langle\mathfrak{b}_{1}, \mathfrak{c}_{1}\left(x_{1}-x, s_{k}^{(1)}, \tau\right)\right\rangle}{\sqrt{s_{k}^{(1)} / \tau d_{1}} \Delta_{1}^{\prime}\left(s_{k}^{(1)}\right)} \\
& \cdot \int_{x_{0}}^{x_{1}} \int_{0}^{t} \frac{e^{s_{k}^{(1)}(t-\varsigma) / \tau} r_{1}(y, \varsigma)\left\langle\mathfrak{a}_{1}, \mathfrak{c}_{1}\left(x_{0}-y, s_{k}^{(1)}, \tau\right)\right\rangle}{\left(\varsigma^{m} / \tau^{m}+\tau^{m}\right)^{\rho}} d \varsigma d y \\
& -\frac{1}{\tau d_{1}} \sum_{k=1}^{\infty} \frac{e^{s_{k}^{(1)} t / \tau}\left\langle\mathfrak{b}_{1}, \mathfrak{c}_{1}\left(x_{1}-x, s_{k}^{(1)}, \tau\right)\right\rangle}{\sqrt{s_{k}^{(1)} / \tau d_{1}} \Delta_{1}^{\prime}\left(s_{k}^{(1)}\right)} \\
& \cdot \int_{x_{0}}^{x_{1}}\left\langle\mathfrak{a}_{1}, \mathfrak{c}_{1}\left(x_{0}-y, s_{k}^{(1)}, \tau\right)\right\rangle \eta_{1}(y) d y \text {, } \\
& \theta_{2}(x, t, \tau)=-\frac{1}{\tau d_{2}} \int_{x_{1}}^{x_{2}} \Theta_{0}^{(2)}\left(x_{1}-x, x_{2}-y\right) \eta_{2}(y) d y \\
& -\frac{1}{\tau d_{2}} \int_{x_{1}}^{x_{2}} \int_{0}^{t} \Theta_{0}^{(2)}\left(x_{1}-x, x_{2}-y\right) \frac{r_{2}(y, \varsigma)}{\left(\varsigma^{m} / \tau^{m}+\tau^{m}\right)^{\rho}} d \varsigma d y \\
& -\frac{1}{\tau d_{2}} \sum_{k=1}^{\infty} \frac{\left\langle\mathfrak{b}_{2}, \mathfrak{c}_{2}\left(x_{2}-x, s_{k}^{(2)}, \tau\right)\right\rangle}{\sqrt{s_{k}^{(2)} / \tau d_{2}} \Delta_{2}^{\prime}\left(s_{k}^{(2)}\right)} \\
& \cdot \int_{x_{1}}^{x_{2}} \int_{0}^{t} \frac{e^{s_{k}^{(2)}(t-\varsigma) / \tau} r_{2}(y, \varsigma)\left\langle\mathfrak{a}_{2}, \mathfrak{c}_{2}\left(x_{1}-y, s_{k}^{(2)}, \tau\right)\right\rangle}{\left(\varsigma^{m} / \tau^{m}+\tau^{m}\right)^{\rho}} d \varsigma d y \\
& -\frac{1}{\tau d_{2}} \sum_{k=1}^{\infty} \frac{e^{s_{k}^{(2)} t / \tau}\left\langle\mathfrak{b}_{2}, \mathfrak{c}_{2}\left(x_{2}-x, s_{k}^{(2)}, \tau\right)\right\rangle}{\sqrt{s_{k}^{(2)} / \tau d_{2}} \Delta_{2}^{\prime}\left(s_{k}^{(2)}\right)} \\
& \cdot \int_{x_{1}}^{x_{2}}\left\langle\mathfrak{a}_{2}, \mathfrak{c}_{2}\left(x_{1}-y, s_{k}^{(2)}, \tau\right)\right\rangle \eta_{2}(y) d y \text {, }
\end{aligned}
$$

where $\Theta_{0}^{(1)}$ and $\Theta_{0}^{(2)}$ can be defined as in Lemma 4 .

Also, similar to (93), with the respective forms $\Phi_{0}^{(1)}$ and $\Phi_{0}^{(2)}$ from Lemma 5 and the matching condition $\xi_{1}(t)=\zeta_{2}$ 
$(t)$, we get

$$
\begin{aligned}
\varphi_{1}(x, t, \tau)= & \lambda(t, \tau) \zeta_{1}(t) \Phi_{0}^{(1)}\left(x_{1}-x ; \mathfrak{b}_{1}\right) \\
& +\sum_{k=1}^{\infty} \frac{\Gamma_{k}^{(1)} \zeta_{1}(t)}{s^{(1)} \Delta_{1}^{\prime}\left(s_{k}^{(1)}\right)}\left\langle\mathfrak{b}_{1}, \mathfrak{c}_{1}\left(x_{1}-x, s_{k}^{(1)}, \tau\right)\right\rangle \\
& -\lambda(t, \tau) \xi_{1}(t) \Phi_{0}^{(1)}\left(x_{0}-x ; \mathfrak{a}_{1}\right) \\
& -\sum_{k=1}^{\infty} \frac{\Gamma_{k}^{(1)} \xi_{1}(t)}{s_{k}^{(1)} \Delta_{1}^{\prime}\left(s_{k}^{(1)}\right)}\left\langle\mathfrak{a}_{1}, \mathfrak{c}_{1}\left(x_{0}-x, s_{k}^{(1)}, \tau\right)\right\rangle \\
& +\theta_{1}(x, t, \tau), \\
\varphi_{2}(x, t, \tau)= & \lambda(t, \tau) \xi_{1}(t) \Phi_{0}^{(2)}\left(x_{2}-x ; \mathfrak{b}_{2}\right) \\
& +\sum_{k=1}^{\infty} \frac{\Gamma_{k}^{(2)} \xi_{1}(t)}{s_{k}^{(2)} \Delta_{2}^{\prime}\left(s_{k}^{(2)}\right)}\left\langle\mathfrak{b}_{2}, \mathfrak{c}_{2}\left(x_{2}-x, s_{k}^{(2)}, \tau\right)\right\rangle \\
& -\lambda(t, \tau) \xi_{2}(t) \Phi_{0}^{(2)}\left(x_{1}-x ; \mathfrak{a}_{2}\right) \\
& -\sum_{k=1}^{\infty} \frac{\Gamma_{k}^{(2)} \xi_{2}(t)}{s_{k}^{(2)} \Delta_{2}^{\prime}\left(s_{k}^{(2)}\right)}\left\langle\mathfrak{a}_{2}, \mathfrak{c}_{2}\left(x_{1}-x, s_{k}^{(2)}, \tau\right)\right\rangle \\
& +\theta_{2}(x, t, \tau),
\end{aligned}
$$

where the operators $\Gamma_{k}^{(1)}$ and $\Gamma_{k}^{(2)}$ are obtained from (94). The matching condition $\varphi_{1}\left(x_{1}, t\right)=\Lambda_{1} \varphi_{2}\left(x_{1}, t\right)$ yields

$$
\begin{aligned}
& \left(\Lambda_{1} \lambda(t, \tau) \Phi_{0}^{(2)}\left(x_{2}-x_{1} ; \mathfrak{b}_{2}\right)+\lambda(t, \tau) \Phi_{0}^{(1)}\left(x_{0}-x_{1} ; \mathfrak{a}_{1}\right)\right) \xi_{1}(t) \\
& +\sum_{k=1}^{\infty}\left\langle\frac{\left\langle\mathfrak{a}_{1}, \mathfrak{c}_{1}\left(x_{0}-x_{1}, s_{k}^{(1)}, \tau\right)\right\rangle}{s_{k}^{(1)} \Delta_{1}^{\prime}\left(s_{k}^{(1)}\right)} \Gamma_{k}^{(1)} \xi_{1}(t)\right. \\
& \left.\quad-\frac{\Lambda_{1}\left\langle\mathfrak{b}_{2}, \mathfrak{c}_{2}\left(x_{2}-x_{1}, s_{k}^{(2)}, \tau\right)\right\rangle}{s_{k}^{(2)} \Delta_{2}^{\prime}\left(s_{k}^{(2)}\right)} \Gamma_{k}^{(2)} \xi_{1}(t)\right) \\
& \quad=\lambda(t, \tau) \zeta_{1}(t) \Phi_{0}^{(1)}\left(0 ; \mathfrak{b}_{1}\right) \quad+\theta_{1}\left(x_{1}, t, \tau\right) \\
& +\sum_{k=1}^{\infty} \frac{\left\langle\mathfrak{b}_{1}, \mathfrak{c}_{1}\left(0, s_{k}^{(1)}, \tau\right)\right\rangle}{s_{k}^{(1)} \Delta_{1}^{\prime}\left(s_{k}^{(1)}\right)} \Gamma_{k}^{(1)} \zeta_{1}(t)+\Lambda_{1} \lambda(t, \tau) \xi_{2}(t) \Phi_{0}^{(2)}\left(0 ; \mathfrak{a}_{2}\right) \\
& +\sum_{k=1}^{\infty} \frac{\Lambda_{1}\left\langle\mathfrak{a}_{2}, \mathfrak{c}_{2}\left(0, s_{k}^{(2)}, \tau\right)\right\rangle}{s_{k}^{(2)} \Delta_{2}^{\prime}\left(s_{k}^{(2)}\right)} \Gamma_{k}^{(2)} \xi_{2}(t)-\Lambda_{1} \theta_{2}\left(x_{1}, t, \tau\right) .
\end{aligned}
$$

For the unknown function $\xi_{1}$, we can rewrite the linear integral equation (127) as

$$
\lambda(t, \tau) \xi_{1}(t)+\sum_{k=1}^{\infty}\left(a_{k} \Gamma_{k}^{(1)} \xi_{1}(t)+b_{k} \Gamma_{k}^{(2)} \xi_{1}(t)\right)=c(t)
$$

where

$$
\begin{aligned}
a_{k}= & \frac{\left\langle\mathfrak{a}_{1}, \mathfrak{c}_{1}\left(x_{0}-x_{1}, s_{k}^{(1)}, \tau\right)\right\rangle}{s_{k}^{(1)} \Delta_{1}{ }^{\prime}\left(s_{k}^{(1)}\right)\left(\Lambda_{1} \Phi_{0}^{(2)}\left(x_{2}-x_{1} ; \mathfrak{b}_{2}\right)+\Phi_{0}^{(1)}\left(x_{0}-x_{1} ; \mathfrak{a}_{1}\right)\right)}, \\
b_{k}= & -\frac{\Lambda_{1}\left\langle\mathfrak{b}_{2}, \mathfrak{C}_{2}\left(x_{2}-x_{1}, s_{k}^{(2)}, \tau\right)\right\rangle}{s_{k}^{(2)} \Delta_{2}{ }^{\prime}\left(s_{k}^{(2)}\right)\left(\Lambda_{1} \Phi_{0}^{(2)}\left(x_{2}-x_{1} ; \mathfrak{b}_{2}\right)+\Phi_{0}^{(1)}\left(x_{0}-x_{1} ; \mathfrak{a}_{1}\right)\right)}, \\
c(t)= & \frac{1}{\Lambda_{1} \Phi_{0}^{(2)}\left(x_{2}-x_{1} ; \mathfrak{b}_{2}\right)+\Phi_{0}^{(1)}\left(x_{0}-x_{1} ; \mathfrak{a}_{1}\right)} \\
& \cdot\left(\lambda(t, \tau) \zeta_{1}(t) \Phi_{0}^{(1)}\left(0 ; \mathfrak{b}_{1}\right)+\theta_{1}\left(x_{1}, t, \tau\right)\right. \\
& +\sum_{k=1}^{\infty} \frac{\left\langle\mathfrak{b}_{1}, \mathfrak{c}_{1}\left(0, s_{k}^{(1)}, \tau\right)\right\rangle}{s_{k}^{(1)} \Delta_{1}^{\prime}\left(s_{k}^{(1)}\right)} \Gamma_{k}^{(1)} \zeta_{1}(t)+\Lambda_{1} \lambda(t, \tau) \xi_{2}(t) \Phi_{0}^{(2)}\left(0 ; \mathfrak{a}_{2}\right) \\
& \left.+\sum_{k=1}^{\infty} \frac{\Lambda_{1}\left\langle\mathfrak{a}_{2}, \mathfrak{c}_{2}\left(0, s_{k}^{(2)}, \tau\right)\right\rangle}{s_{k}^{(2)} \Delta_{2}^{\prime}\left(s_{k}^{(2)}\right)} \Gamma_{k}^{(2)} \xi_{2}(t)-\Lambda_{1} \theta_{2}\left(x_{1}, t, \tau\right)\right) .
\end{aligned}
$$

Inspired by the convolution formula (31), the natural transform of (128) is

$$
\begin{aligned}
& \mathbb{N}\left[\xi_{1}(t) \lambda(t, \tau)\right](s, \tau) \\
& +\sum_{k=1}^{\infty}\left(a_{k}\left(\mathbb{N}\left[\xi_{1}(t) \lambda(t, \tau)\right](s, \tau)-\frac{\tau s_{k}^{(1)}}{s-s_{k}^{(1)}} \mathbb{N}\left[\xi_{1}(t) \lambda(t, \tau)\right]\right)\right. \\
& \left.+b_{k}\left(\mathbb{N}\left[\xi_{1}(t) \lambda(t, \tau)\right](s, \tau)-\frac{\tau s_{k}^{(2)}}{s-s_{k}^{(2)}} \mathbb{N}\left[\xi_{1}(t) \lambda(t, \tau)\right]\right)\right) \\
& \quad=\mathbb{N}[c(t)](s, \tau),
\end{aligned}
$$

which can be rewritten as

$$
(1-\mathbb{N}[\psi(t)](s, \tau)) \mathbb{N}\left[\xi_{1}(t) \lambda(t, \tau)\right](s, \tau)=\mathbb{N}[c(t)](s, \tau) .
$$

That is,

$$
\begin{aligned}
\mathbb{N} & {\left[\xi_{1}(t) \lambda(t, \tau)\right](s, \tau) } \\
& =\frac{1}{(1-\mathbb{N}[\psi(t)](s, \tau))}\{\mathbb{N}[c(t)](s, \tau)\} \\
& =\left(1+\mathbb{N}[\psi(t)](s, \tau)+(\mathbb{N}[\psi(t)](s, \tau))^{2}+\cdots\right)\{\mathbb{N}[c(t)](s, \tau)\} \\
& =\mathbb{N}[c(t)](s, \tau)+\mathbb{N}[c(t)](s, \tau) \sum_{m=1}^{\infty}(\mathbb{N}[\psi(t)](s, \tau))^{m},
\end{aligned}
$$


where

$\mathbb{N}[\psi(t)](s, \tau)=-\sum_{k=1}^{\infty}\left(a_{k}\left(1-\frac{\tau s_{k}^{(1)}}{s-s_{k}^{(1)}}\right)+b_{k}\left(1-\frac{\tau s_{k}^{(2)}}{s-s_{k}^{(2)}}\right)\right)$,

for which the inverse natural transform is

$$
\begin{aligned}
\psi(t)= & -\tau \sum_{k=1}^{\infty}\left(a_{k}+b_{k}\right) \delta_{0}(t) \\
& +\tau \sum_{k=1}^{\infty}\left(a_{k} s_{k}^{(1)} e^{\left(s_{k}^{(1)} / \tau\right) t}+b_{k} s_{k}^{(2)} e^{\left(s_{k}^{(2)} / \tau\right) t}\right)
\end{aligned}
$$

where $\delta_{0}$ is the Dirac delta function. Hence, we have

$$
\xi_{1}(t)=\frac{1}{\lambda(t, \tau)}\left(c(t)+\sum_{m=1}^{\infty} \psi_{m} * c(t)\right),
$$

where for $m \geq 2, \psi_{m}$ is the $m$-times self-convolution of $\psi$. Thus, one can conclude the solution to the bilayer diffusion problem (112)-(119) by the formulas (125) and (126), together with (123) and (124), with $\xi_{1}$ given in (135). Now, it is time to attack an illustrative example in the following subsection.

3.1.1. Illustrative Example. Here, we discuss the solvability of the following two-layer diffusion system.

Example 3. Temperature distribution in the two-layer slab with mixed boundary condition.

Consider the following initial boundary value system. The diffusion equations are

$$
\frac{\partial \varphi_{k}}{\partial t}=d_{k} \frac{\partial^{2} \varphi_{k}}{\partial x^{2}}, \quad x_{k-1}<x<x_{k}, x_{0}=0, t>0, k=1,2,
$$

the initial conditions are

$$
\varphi_{k}(x, 0)=\eta_{k}(x), \quad x_{k-1} \leq x \leq x_{k}, k=1,2,
$$

the outer boundary conditions are

$$
\varphi_{1}(0, t)=0, \frac{\partial \varphi_{2}\left(x_{2}, t\right)}{\partial x}=0, \quad t \geq 0
$$

and the interface conditions at $x=x_{1}$ are

$$
\varphi_{1}\left(x_{1}, t\right)=\varphi_{2}\left(x_{1}, t\right), d_{1} \frac{\partial \varphi_{1}\left(x_{1}, t\right)}{\partial x}=d_{2} \frac{\partial \varphi_{2}\left(x_{1}, t\right)}{\partial x}, \quad t \geq 0 .
$$

Comparing this problem with the general one that is defined by (112)-(119) reveals

$$
\begin{gathered}
\mathfrak{a}_{1}=(1,0), \mathfrak{b}_{1}=\left(0, d_{1}\right), \mathfrak{a}_{2}=\left(0, d_{2}\right), \mathfrak{b}_{2}=(0,1), \\
r_{1}(x, t)=r_{2}(x, t)=\zeta_{1}(t)=\xi_{2}(t)=0, \xi_{1}(t)=\zeta_{2}(t) .
\end{gathered}
$$

Here, we will consider $\rho=0$ and $\tau=1$.

Then, the solution of the problem (136)-(139) can be obtained from (125) and (126) as

$$
\begin{aligned}
& \varphi_{1}(x, t)=-\xi_{1}(t) \Phi_{0}^{(1)}\left(x_{0}-x ; \mathfrak{a}_{1}\right)
\end{aligned}
$$

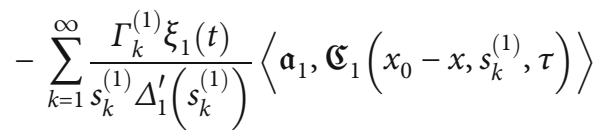

$$
\begin{aligned}
& +\theta_{1}(x, t) \text {, }
\end{aligned}
$$

$$
\begin{aligned}
\varphi_{2}(x, t)= & \xi_{1}(t) \Phi_{0}^{(2)}\left(x_{2}-x ; \mathfrak{b}_{2}\right) \\
& +\sum_{k=1}^{\infty} \frac{\Gamma_{k}^{(2)} \xi_{1}(t)}{s_{k}^{(2)} \Delta_{2}^{\prime}\left(s_{k}^{(2)}\right)}\left\langle\mathfrak{b}_{2}, \mathfrak{c}_{2}\left(x_{2}-x, s_{k}^{(2)}, \tau\right)\right\rangle \\
& +\theta_{2}(x, t),
\end{aligned}
$$

with

$$
\begin{aligned}
\theta_{1}(x, t)= & -\frac{1}{d_{1}} \int_{x_{0}}^{x_{1}} \Theta_{0}^{(1)}\left(x_{0}-x, x_{1}-y\right) \eta_{1}(y) d y \\
& -\frac{1}{d_{1}} \sum_{k=1}^{\infty} \frac{e^{s_{k}^{(1)}} t\left\langle b_{1}, \mathfrak{C}_{1}\left(x_{1}-x, s_{k}^{(1)}\right)\right\rangle}{\sqrt{s_{k}^{(1)} / d_{1}} \Delta_{1}^{\prime}\left(s_{k}^{(1)}\right)} \\
& \cdot \int_{x_{0}}^{x_{1}}\left\langle a_{1}, \mathfrak{c}_{1}\left(x_{0}-y, s_{k}^{(1)}\right)\right\rangle \eta_{1}(y) d y, \\
\theta_{2}(x, t)= & -\frac{1}{d_{2}} \int_{x_{1}}^{x_{2}} \Theta_{0}^{(2)}\left(x_{1}-x, x_{2}-y\right) \eta_{2}(y) d y \\
& -\frac{1}{d_{2}} \sum_{k=1}^{\infty} \frac{e^{s_{k}^{(2)}} t\left\langle b_{2}, \mathfrak{c}_{2}\left(x_{2}-x, s_{k}^{(2)}\right)\right\rangle}{\sqrt{s_{k}^{(2)} / d_{2}} \Delta_{2}^{\prime}\left(s_{k}^{(2)}\right)} \\
& \cdot \int_{x_{1}}^{x_{2}}\left\langle a_{2}, \mathfrak{c}_{2}\left(x_{1}-y, s_{k}^{(2)}\right)\right\rangle \eta_{2}(y) d y .
\end{aligned}
$$

The operators $\Gamma_{k}^{(1)}$ and $\Gamma_{k}^{(2)}$ are obtained from (94). 
Next, we are going to simplify these formulas. Direct computations give

$$
\begin{gathered}
\Delta_{1}(s)=d_{1} \sqrt{\frac{s}{d_{1}}} \cosh \sqrt{\frac{s}{d_{1}}} x_{1}, \Delta_{2}(s)=-d_{2} \frac{s}{d_{2}} \sinh \sqrt{\frac{s}{d_{2}}}\left(x_{2}-x_{1}\right), \\
\Delta_{1}^{\prime}(s)=d_{1}\left[\frac{x_{1}}{2 d_{1}} \sinh \sqrt{\frac{s}{d_{1}}} x_{1}+\frac{1}{2 \sqrt{d_{1} s}} \cosh \sqrt{\frac{s}{d_{1}}} x_{1}\right], \\
\Delta_{2}^{\prime}(s)=-d_{2}\left[\frac{1}{d_{2}} \sinh \sqrt{\frac{s}{d_{2}}}\left(x_{2}-x_{1}\right)+\frac{x_{2}-x_{1}}{2 d_{2}} \sqrt{\frac{s}{d_{2}}} \cosh \sqrt{\frac{s}{d_{2}}}\left(x_{2}-x_{1}\right)\right] .
\end{gathered}
$$

It is clear that $\Delta_{1}\left(s_{k}^{(1)}\right)=0$ and $\Delta_{2}\left(s_{k}^{(2)}\right)=0$ when

$$
s_{k}^{(1)}=\frac{(2 k-1)^{2} \pi^{2} d_{1}}{4 x_{1}^{2}}, s_{k}^{(2)}=-\frac{k^{2} \pi^{2} d_{2}}{\left(x_{2}-x_{1}\right)^{2}}, \quad k=1,2,3, \cdots,
$$

respectively. Moreover,

$$
\begin{aligned}
& \frac{\left\langle\mathfrak{a}_{1}, \mathfrak{c}_{1}(x ; s)\right\rangle\left\langle\mathfrak{b}_{1}, \mathfrak{c}_{1}(y ; s)\right\rangle}{\sqrt{s / d_{1}} \Delta_{1}(s)}=\frac{\sinh \sqrt{s / d_{1}} x \cosh \sqrt{s / d_{1}} y}{\sqrt{s / d_{1}} \cosh \sqrt{s / d_{1}} x_{1}}=O(1), \\
& \frac{\left\langle\mathfrak{a}_{2}, \mathfrak{c}_{2}(x ; s)\right\rangle\left\langle\mathfrak{b}_{2}, \mathfrak{c}_{2}(y ; s)\right\rangle}{\sqrt{s / d_{2}} \Delta_{2}(s)}=-\frac{\cosh \sqrt{s / d_{2}} x \cosh \sqrt{s / d_{2}} y}{\sqrt{s / d_{2}} \sinh \sqrt{s / d_{2}}\left(x_{2}-x_{1}\right)}=O\left(\frac{1}{s}\right),
\end{aligned}
$$

as $s \longrightarrow 0$. Therefore, $(70)$ gives $\Theta_{0}^{(1)}(x, y)=0$ and $\Theta_{0}^{(2)}(x, y)$ $=-\left(d_{2} /\left(x_{2}-x_{1}\right)\right)$.

Further,

$$
\begin{gathered}
\left\langle\mathfrak{a}_{1}, \mathfrak{c}_{1}\left(-y ; s_{k}^{(1)}\right)\right\rangle=-i \sin \frac{(2 k-1) \pi}{2 x_{1}} y, \\
\left\langle\mathfrak{b}_{1}, \mathfrak{c}_{1}\left(x_{1}-x ; s_{k}^{(1)}\right)\right\rangle=(-1)^{k+1} \frac{(2 k-1) \pi d_{1}}{2 x_{1}} i \sin \frac{(2 k-1) \pi}{2 x_{1}} x, \\
\sqrt{\frac{s_{k}^{(1)}}{d_{1}} \Delta_{1}^{\prime}\left(s_{k}^{(1)}\right)=(-1)^{k} \frac{(2 k-1) \pi}{4}, \quad k=1,2,3, \cdots,} \\
\left\langle\mathfrak{a}_{2}, \mathfrak{c}_{2}\left(x_{1}-y ; s_{k}^{(2)}\right)\right\rangle=\frac{k \pi d_{2} i}{x_{2}-x_{1}} \cos \frac{k \pi}{x_{2}-x_{1}}\left(x_{1}-y\right), \\
\left\langle\mathfrak{b}_{2}, \mathfrak{c}_{2}\left(x_{2}-x ; s_{k}^{(2)}\right)\right\rangle=\frac{k \pi i}{x_{2}-x_{1}} \cos \frac{k \pi}{x_{2}-x_{1}}\left(x_{2}-x\right), \\
\sqrt{\frac{s_{k}^{(2)}}{d_{2}} \Delta_{2}^{\prime}\left(s_{k}^{(2)}\right)}=(-1)^{k} \frac{k^{2} \pi^{2}}{2\left(x_{2}-x_{1}\right)} .
\end{gathered}
$$

Thus,

$$
\begin{aligned}
\theta_{1}(x, t)= & \sum_{k=1}^{\infty}\left[\frac{2}{x_{1}} \int_{0}^{x_{1}} \eta_{1}(y) \sin \frac{(2 k-1) \pi y}{2 x_{1}} d y\right] e^{-\left((2 k-1)^{2} \pi^{2} / 4 x_{1}^{2}\right) d_{1} t} \\
& \cdot \sin \frac{(2 k-1) \pi}{2 x_{1}} x, \\
\theta_{2}(x, t)= & -\frac{1}{x_{2}-x_{1}} \int_{x_{1}}^{x_{2}} \eta_{2}(y) d y+\sum_{k=1}^{\infty}(-1)^{k} \\
& \cdot\left[\frac{1}{x_{2}-x_{1}} \int_{x_{1}}^{x_{2}} \eta_{2}(y) \cos \frac{k \pi}{x_{2}-x_{1}}\left(x_{1}-y\right) d y\right] \\
& \cdot e^{-\left(k^{2} \pi^{2}\right)^{2} \pi^{2} /\left(x_{2}-x_{1}\right)^{2} d_{2} t} \cos \frac{k \pi}{x_{2}-x_{1}}\left(x_{2}-x_{1}\right) .
\end{aligned}
$$

Similarly, we have

$$
\begin{gathered}
\frac{\left\langle\mathfrak{a}_{1}, \mathfrak{c}_{1}\left(x_{0}-x, s\right)\right\rangle}{s \Delta_{1}(s)}=\frac{\sinh \sqrt{s / d_{1}}\left(x_{0}-x\right)}{s d_{1} \sqrt{s / d_{1}} \cosh \sqrt{s / d_{1}} x_{1}}=O\left(\frac{1}{s}\right), \\
\frac{\left\langle\mathfrak{b}_{2}, \mathfrak{c}_{2}\left(x_{2}-x, s\right)\right\rangle}{s \Delta_{2}(s)}=-\frac{\sqrt{s / d_{2}} \cosh \sqrt{s / d_{2}}\left(x_{2}-x\right)}{s d_{2}\left(s / d_{1}\right) \sinh \sqrt{s / d_{2}}\left(x_{2}-x_{1}\right)}=O\left(\frac{1}{s^{2}}\right) .
\end{gathered}
$$

So, (88) gives

$$
\begin{aligned}
\Phi_{0}^{(1)}\left(x_{0}-x ; \mathfrak{a}_{1}\right) & =-\frac{x}{d_{1}}, \Phi_{0}^{(2)}\left(x_{2}-x ; \mathfrak{b}_{2}\right) \\
& =\frac{1}{6 d_{2}\left(x_{2}-x_{1}\right)}\left\{3\left(x_{2}-x\right)^{2}-\left(x_{2}-x_{1}\right)^{2}\right\}
\end{aligned}
$$

Hence, loading the quantities in (141) and (142) gives the solution to problem (136)-(139) as

$$
\begin{aligned}
\varphi_{1}(x, t)= & \frac{x}{d_{1}} \xi_{1}(t) \\
& +\frac{8 x_{1}}{\pi^{2} d_{1}} \sum_{k=1}^{\infty} \frac{(-1)^{k} \Gamma_{k}^{(1)} \xi_{1}(t)}{(2 k-1)^{2}} \sin \frac{(2 k-1) \pi}{2 x_{1}} x+\theta_{1}(x, t), \\
\varphi_{2}(x, t)= & \frac{3\left(x_{2}-x\right)^{2}-\left(x_{2}-x_{1}\right)^{2}}{6 d_{2}\left(x_{2}-x_{1}\right)} \xi_{1}(t) \\
+ & \frac{2\left(x_{2}-x\right)}{d_{2} \pi^{2}} \sum_{k=1}^{\infty} \frac{(-1)^{k} \Gamma_{k}^{(2)} \xi_{1}(t)}{k^{2}} \cos \frac{k \pi}{x_{2}-x_{1}}\left(x_{2}-x\right)+\theta_{2}(x, t) .
\end{aligned}
$$

Applying the matching condition $\varphi_{1}\left(x_{1}, t\right)=\varphi_{2}\left(x_{1}, t\right)$ gives

$$
\xi_{1}(t)+\sum_{k=1}^{\infty}\left[a_{k} \Gamma_{k}^{(1)} \xi_{1}(t)+b_{k} \Gamma_{k}^{(2)} \xi_{1}(t)\right]=c(t),
$$


where

$$
\begin{gathered}
a_{k}=-\frac{24 d_{2} x_{1}}{(2 k-1)^{2} \pi^{2}\left[\left(3 d_{2}-d_{1}\right) x_{1}+d_{1} x_{2}\right]}, \\
b_{k}=-\frac{6 d_{1}\left(x_{2}-x_{1}\right)}{k^{2} \pi^{2}\left[\left(3 d_{2}-d_{1}\right) x_{1}+d_{1} x_{2}\right]}, \\
c(t)=\frac{3 d_{1} d_{2}}{\left(3 d_{2}-d_{1}\right) x_{1}+d_{1} x_{2}}\left[\theta_{1}\left(x_{1}, t\right)-\theta_{2}\left(x_{1}, t\right)\right] .
\end{gathered}
$$

To solve this integral equation, we use (134), to obtain

$$
\begin{aligned}
\psi(t)= & \delta_{0}(t)-\frac{6 d_{1} d_{2}}{\left(3 d_{2}-d_{1}\right) x_{1}+d_{1} x_{2}} \sum_{k=1}^{\infty} \\
& \cdot\left(\frac{1}{x_{1}} e^{-\left((2 k-1)^{2} \pi^{2} d_{1} / 4 x_{1}^{2}\right) t}+\frac{1}{x_{2}-x_{1}} e^{-\left(k^{2} \pi^{2} d_{2} /\left(x_{2}-x_{1}\right)^{2}\right) t}\right),
\end{aligned}
$$

where $\delta_{0}$ is the Dirac delta function. Here, we used

$$
\sum_{k=1}^{\infty} \frac{1}{(2 k-1)^{2}}=\frac{\pi^{2}}{8}, \sum_{k=1}^{\infty} \frac{1}{k^{2}}=\frac{\pi^{2}}{6} .
$$

Hence, from (135), we have

$$
\begin{aligned}
\xi_{1}(t)= & c(t)+(\psi * c)(t)+(\psi * \psi * c)(t)+\cdots=c(t) \\
& +\sum_{m=1}^{\infty}\left(\psi_{m} * c\right)(t) .
\end{aligned}
$$

3.2. Solution of a Multilayer Problem. Here, we investigate the solvability of the main problem (2)-(8), through solving the initial boundary value problems (9)-(11). Similar to what we have denoted in Section 2, we consider the following notations:

$$
\begin{gathered}
\mathfrak{a}_{j}= \begin{cases}(l, \iota), & j=1, \\
\left(v_{j}, \mu_{j}\right), & 2 \leq j \leq n,\end{cases} \\
\mathfrak{b}_{j}= \begin{cases}\left(v_{j}, \mu_{j}\right), & 1 \leq j \leq n-1, \\
(\ell, l), & j=n,\end{cases}
\end{gathered}
$$

and, for all $1 \leq j \leq n$,

$$
\begin{aligned}
& \mathfrak{\Omega}_{j}(y ; s, \tau)=\left(\cosh \sqrt{\frac{s}{\tau d_{j}}} y, \sqrt{\frac{s}{\tau d_{j}}} \sinh \sqrt{\frac{s}{\tau d_{j}} y}\right), \\
& \mathfrak{c}_{j}(y ; s, \tau)=\left(\sinh \sqrt{\frac{s}{\tau d_{j}} y} y \sqrt{\frac{s}{\tau d_{j}}} \cosh \sqrt{\frac{s}{\tau d_{j}} y}\right) .
\end{aligned}
$$

Moreover, we define

$$
\begin{aligned}
\Delta_{j}(s)= & \left\langle\mathfrak{b}_{j}, \mathfrak{c}_{j}\left(x_{j}, s, \tau\right)\right\rangle\left\langle\mathfrak{a}_{j}, \mathfrak{\mathfrak { I }}_{j}\left(x_{j-1}, s, \tau\right)\right\rangle \\
& -\left\langle\mathfrak{a}_{j}, \mathfrak{c}_{j}\left(x_{j-1}, s, \tau\right)\right\rangle\left\langle\mathfrak{b}_{j}, \mathfrak{Q}_{j}\left(x_{j}, s, \tau\right)\right\rangle,
\end{aligned}
$$

and let $\left\{s_{k}^{(j)}\right\}_{k=1}^{\infty}$ be the sequence of zeros of the function $\Delta_{j}$ (s) for all $1 \leq j \leq n$, i.e.,

$$
\Delta_{j}\left(s_{k}^{(j)}\right)=0, \Delta_{j}^{\prime}\left(s_{k}^{(j)}\right) \neq 0 \quad(k=1,2, \cdots) .
$$

Analogue to the computations of (84) and (93), we have for the current case, for all $j=1, \cdots, n$,

$$
\begin{aligned}
& \theta_{j}(x, t, \tau)=-\frac{1}{\tau d_{j}} \int_{x_{j-1}}^{x_{j}} \Theta_{0}^{(j)}\left(x_{j-1}-x, x_{j}-y\right) \eta_{j}(y) d y \\
& -\frac{1}{\tau d_{j}} \int_{x_{j-1}}^{x_{j}} \int_{0}^{t} \Theta_{0}^{(j)}\left(x_{j-1}-x, x_{j}-y\right) \frac{r_{j}(y, \zeta)}{\left(\zeta^{m} / \tau^{m}+\tau^{m}\right)^{\rho}} d \zeta d y \\
& -\frac{1}{\tau d_{j}} \sum_{k=1}^{\infty} \frac{\left\langle\mathfrak{b}_{j}, \mathfrak{c}_{j}\left(x_{j}-x, s_{k}^{(j)}, \tau\right)\right\rangle}{\sqrt{s_{k}^{(j)} / \tau d_{j}} \Delta_{j}^{\prime}\left(s_{k}^{(j)}\right)} \\
& \cdot \int_{x_{j-1}}^{x_{j}} \int_{0}^{t} \frac{e^{s_{k}^{(j)}(t-\zeta) / \tau} r_{j}(y, \zeta)\left\langle\mathfrak{a}_{j}, \mathfrak{c}_{j}\left(x_{j-1}-y, s_{k}^{(j)}, \tau\right)\right\rangle}{\left(\zeta^{m} / \tau^{m}+\tau^{m}\right)^{\rho}} d \zeta d y \\
& -\frac{1}{\tau d_{j}} \sum_{k=1}^{\infty} \frac{e^{s_{k}^{(j)} t / \tau}\left\langle\mathfrak{b}_{j}, \mathfrak{c}_{j}\left(x_{j}-x, s_{k}^{(j)}, \tau\right)\right\rangle}{\sqrt{s_{k}^{(j)} / \tau d_{j}} \Delta_{j}^{\prime}\left(s_{k}^{(j)}\right)} \\
& \cdot \int_{x_{j-1}}^{x_{j}}\left\langle\mathfrak{a}_{j}, \mathfrak{c}_{j}\left(x_{j-1}-y, s_{k}^{(j)}, \tau\right)\right\rangle \eta_{j}(y) d y,
\end{aligned}
$$

where $\Theta_{0}^{(j)}$ can be defined in a similar way as in Lemma 4 , and

$$
\begin{aligned}
\varphi_{j}(x, t, \tau)= & \lambda(t, \tau) \zeta_{j}(t) \Phi_{j}\left(x_{j}-x, 0 ; \mathfrak{b}_{j}\right) \\
& -\int_{0}^{t} \lambda(\varsigma, \tau) D_{2} \Phi_{j}\left(x_{j}-x, t-\varsigma ; \mathfrak{b}_{j}\right) \zeta_{j}(\varsigma) d \varsigma \\
& -\lambda(t, \tau) \xi_{j}(t) \Phi_{j}\left(x_{j-1}-x, 0 ; \mathfrak{a}_{j}\right) \\
& +\int_{0}^{t} \lambda(\varsigma, \tau) D_{2} \Phi_{j}\left(x_{j-1}-x, t-\varsigma ; \mathfrak{a}_{j}\right) \xi_{j}(\varsigma) d \varsigma \\
& +\theta_{j}(x, t, \tau),
\end{aligned}
$$

with the respective forms $\Phi_{j}$ defined by (87) in Lemma 5 . This last equation (162) can be rewritten as

$$
\varphi_{j}(x, t, \tau)=T_{j} \tilde{\zeta}_{j}\left(x_{j}-x, t ; \mathfrak{b}_{j}\right)-T_{j} \tilde{\xi}_{j}\left(x_{j-1}-x, t ; \mathfrak{a}_{j}\right)+\theta_{j}(x, t, \tau),
$$

in which $\tilde{\zeta}_{j}=\lambda(t, \tau) \zeta_{j}(t), \tilde{\xi}_{j}=\lambda(t, \tau) \xi_{j}(t)$ and the linear 
operator $T_{j}$ is defined by

$$
T_{j} \varphi(y, t ; \mathbf{L})=\Phi_{j}(y, 0 ; \mathbf{L}) \varphi(t)-\int_{0}^{t} D_{2} \Phi_{j}(y, t-\varsigma ; \mathbf{L}) \varphi(\varsigma) d \varsigma,
$$

for all $j=1, \cdots, n, \mathbf{L} \in \mathbb{R}^{2}$.

The matching conditions $\varphi_{j}\left(x_{j}, t, \tau\right)=\Lambda_{j} \varphi_{j+1}\left(x_{j}, t, \tau\right), j$ $=1, \cdots, n-1$, lead to

$$
\begin{aligned}
T_{j} \tilde{\zeta}_{j}\left(0, t ; \mathfrak{b}_{j}\right)- & T_{j} \tilde{\xi}_{j}\left(x_{j-1}-x_{j}, t ; \mathfrak{a}_{j}\right)+\theta_{j}\left(x_{j}, t, \tau\right) \\
& =\Lambda_{j}\left(T_{j+1} \tilde{\zeta}_{j+1}\left(x_{j+1}-x_{j}, t ; \mathfrak{b}_{j+1}\right)\right. \\
& \left.-T_{j+1} \tilde{\xi}_{j+1}\left(0, t ; \mathfrak{a}_{j+1}\right)+\theta_{j+1}\left(x_{j}, t, \tau\right)\right) .
\end{aligned}
$$

Using the matching conditions (12), we have $\tilde{\zeta}_{j+1}(t)=$ $\tilde{\xi}_{j}(t)$ for all $1 \leq j \leq n-1$. Thus, for $j=1$,

$$
\begin{aligned}
-T_{1} \tilde{\xi}_{1}\left(x_{0}-x_{1}, t ; \mathfrak{a}_{1}\right) & -\Lambda_{1} T_{2} \tilde{\xi}_{1}\left(x_{2}-x_{1}, t ; \mathfrak{b}_{2}\right) \\
& +\Lambda_{1} T_{2} \tilde{\xi}_{2}\left(0, t ; \mathfrak{a}_{2}\right)=\Lambda_{1} \theta_{2}\left(x_{1}, t, \tau\right) \\
& -\theta_{1}\left(x_{1}, t, \tau\right)-T_{1} \tilde{\zeta}_{1}\left(0, t ; \mathfrak{b}_{1}\right) .
\end{aligned}
$$

For $2 \leq j \leq n-2$,

$$
\begin{aligned}
T_{j} \tilde{\xi}_{j-1}\left(0, t ; \mathfrak{b}_{\mathrm{j}}\right) & -T_{\mathrm{j}} \tilde{\xi}_{\mathrm{j}}\left(x_{\mathrm{j}-1}-x_{\mathrm{j}}, t ; \mathfrak{a}_{\mathrm{j}}\right) \\
& -\Lambda_{\mathrm{j}} T_{\mathrm{j}+1} \tilde{\xi}_{\mathrm{j}}\left(x_{j+1}-x_{j}, t ; \mathfrak{b}_{\mathrm{j}+1}\right) \\
& +\Lambda_{\mathrm{j}} T_{j+1} \tilde{\xi}_{j+1}\left(0, t ; \mathfrak{a}_{\mathrm{j}+1}\right)=\Lambda_{\mathrm{j}} \theta_{j+1}\left(x_{\mathrm{j}}, t, \tau\right) \\
& -\theta_{j}\left(x_{j}, t, \tau\right) .
\end{aligned}
$$

$$
\begin{aligned}
& \text { For } j=n-1, \\
& \begin{aligned}
& T_{n-1} \tilde{\xi}_{n-2}\left(0, t ; \mathfrak{b}_{n-1}\right)-T_{n-1} \tilde{\xi}_{n-1}\left(x_{n-2}-x_{n-1}, t ; \mathfrak{a}_{n-1}\right) \\
& \quad-\Lambda_{n-1} T_{n} \tilde{\xi}_{n-1}\left(x_{n}-x_{n-1}, t ; \mathfrak{b}_{n}\right)=\Lambda_{n-1} \theta_{n}\left(x_{n-1}, t, \tau\right) \\
& \quad-\theta_{n-1}\left(x_{n-1}, t, \tau\right)-\Lambda_{n-1} T_{n} \tilde{\xi}_{n}\left(0, t ; \mathfrak{a}_{n}\right) .
\end{aligned}
\end{aligned}
$$

System (166), (167), and (168), of ( $n-1)$ integral equations of the unknowns $\tilde{\xi}_{j} ; 1 \leq j \leq n-1$, can be adjusted as a matrix equation

$$
\mathscr{A}(0) \mathbf{h}(t)+\left(\mathscr{A}^{\prime} * \mathbf{h}\right)(t)=\mathbf{b}(t)
$$

with $\mathscr{A}(t)$ is a tridiagonal matrix of order $n-1$ whose entries are as follows:

$$
\begin{gathered}
-\Phi_{j}\left(x_{j-1}-x_{j}, t ; \mathfrak{a}_{j}\right)-\Lambda_{j} \Phi_{j}\left(x_{j+1}-x_{j}, t ; \mathfrak{b}_{j+1}\right), \quad 1 \leqslant j \leqslant n-1 \text { (main diagonal) } \\
\Lambda_{j} \Phi_{j+1}\left(0, t ; \mathfrak{a}_{j+1}\right), \quad 1 \leqslant j \leqslant n-2 \text { (super diagonal) } \\
\Phi_{j}\left(0, t ; \mathfrak{b}_{j}\right), \quad 2 \leqslant j \leqslant n-1 \text { (subdiagonal), }
\end{gathered}
$$

and the vectors $\mathbf{h}(t)$ and $\mathbf{b}(t)$ are defined as

$$
\begin{gathered}
\mathbf{h}(t)=\left(\begin{array}{c}
\tilde{\xi}_{1}(t) \\
\vdots \\
\tilde{\xi}_{n-1}(t)
\end{array}\right) \\
\mathbf{b} t)=\left(\begin{array}{c}
\Lambda_{1} \theta_{2}\left(x_{1}, t, \tau\right)-\theta_{1}\left(x_{1}, t, \tau\right)-T_{1} \tilde{\zeta}_{1}\left(0, t ; \mathfrak{b}_{1}\right) \\
\Lambda_{2} \theta_{3}\left(x_{2}, t, \tau\right)-\theta_{2}\left(x_{2}, t, \tau\right) \\
\vdots \\
\Lambda_{n-2} \theta_{n-1}\left(x_{n-2}, t, \tau\right)-\theta_{n-2}\left(x_{n-2}, t, \tau\right) \\
\Lambda_{n-1} \theta_{n}\left(x_{n-1}, t, \tau\right)-\theta_{n-1}\left(x_{n-1}, t, \tau\right)-\Lambda_{n-1} T_{n} \tilde{\xi}_{n}\left(0, t ; \mathfrak{a}_{n}\right)
\end{array}\right) .
\end{gathered}
$$

In fact, we can rewrite (169) as

$$
\mathbf{h}(t)=\mathscr{C}(t)+(\mathscr{B} * \mathbf{h})(t),
$$

with $\mathscr{C}(t)=\mathscr{A}(0)^{-1} \mathbf{b}(t)$ and $\mathscr{B}(t)=-\mathscr{A}(0)^{-1} \mathscr{A}^{\prime}(t)$. In view of the convolution formula (31), the natural transform of (172) reads

$$
\mathbb{N}[\mathbf{h}(t)](s, \tau)=\mathbb{N}[\mathscr{C}(t)](s, \tau)+\tau \mathbb{N}[\mathscr{B}(t)](s, \tau) \mathbb{N}[\mathbf{h}(t)](s, \tau),
$$

which is equivalent to

$$
\begin{aligned}
\mathbb{N}[\mathbf{h}(t)](s, \tau)= & (I-\tau \mathbb{N}[\mathscr{B}(t)](s, \tau))^{-1} \mathbb{N}[\mathscr{C}(t)](s, \tau) \\
= & (I+\tau \mathbb{N}[\mathscr{B}(t)](s, \tau) \\
& \left.+(\tau \mathbb{N}[\mathscr{B}(t)](s, \tau))^{2}+\cdots\right) \mathbb{N}[\mathscr{C}(t)](s, \tau),
\end{aligned}
$$

where $I$ is the $(n-1) \times(n-1)$ identity matrix. Once again, throughout the convolution sense (31), the natural transform inversion of (174) is

$$
\begin{aligned}
\mathbf{h}(t)= & \mathscr{C}(t)+(\mathscr{B} * \mathscr{C})(t)+(\mathscr{B} * \mathscr{B} * \mathscr{C})(t)+\cdots=\mathscr{C}(t) \\
& +\sum_{m=1}^{\infty}\left(\mathscr{B}_{m} * \mathscr{C}\right)(t),
\end{aligned}
$$

where $\mathscr{B}_{m}$ is the $m$-times self-convolution of $\mathscr{B}$. Finally, the solution of the nonhomogeneous multilayer diffusion systems (9)-(11) and hence that of the main problem (2)-(8) 
is concluded as

$$
\begin{aligned}
\varphi_{j}(x, t, \tau)= & \lambda(t, \tau) \zeta_{j}(t) \Phi_{0}^{(j)}\left(x_{j}-x ; \mathfrak{b}_{j}\right) \\
& -\lambda(t, \tau) \xi_{j}(t) \Phi_{0}^{(j)}\left(x_{j-1}-x ; \mathfrak{a}_{j}\right) \\
& +\sum_{k=1}^{\infty} \frac{\lambda(t, \tau) \zeta_{j}(t)-\int_{0}^{t} \lambda(\varsigma, \tau) s_{k}^{(j)} e^{s_{k}^{(j)}(t-\varsigma) / \tau} \zeta_{j}(\varsigma) d \varsigma}{s_{k}^{(j)} \Delta_{j}^{\prime}\left(s_{k}^{(j)}\right)} \\
& \cdot\left\langle\mathfrak{b}_{j}, \boldsymbol{c}_{j}\left(x_{j}-x, s_{k}^{(j)}, \tau\right)\right\rangle \\
& -\sum_{k=1}^{\infty} \frac{\lambda(t, \tau) \xi_{j}(t)-\int_{0}^{t} \lambda(\varsigma, \tau) s_{k}^{(j)} e^{s_{k}^{(j)}(t-\varsigma) / \tau} \xi_{j}(\varsigma) d \varsigma}{s_{k}^{(j)} \Delta_{j}^{\prime}\left(s_{k}^{(j)}\right)} \\
& \cdot\left\langle\mathfrak{a}_{j}, \boldsymbol{c}_{j}\left(x_{j-1}-x, s_{k}^{(j)}, \tau\right)\right\rangle+\theta_{j}(x, t, \tau),
\end{aligned}
$$

with the respective forms $\Phi_{0}^{(j)}$ and $\theta_{j}$ defined as in (88) and (161), respectively, for all $j=1, \cdots, n$.

\section{Conclusion}

Throughout the current contribution, a one-dimensional $n$ -layer nonhomogeneous diffusion problem with timevarying data and general interface conditions has been concluded by means of a generalized integral transform. Although most of the previous works have been focused on solving the problems of the homogeneous diffusion equation, the nonhomogeneous diffusion equation problem arises in many physical applications. We have obtained the exact solutions for one- and multilayer nonhomogeneous diffusion problems. The former case has been solved by a new generalized integral transform; the later one (n-layer problem) has been recast in a sequence of one-layer problems. The obtained results generalize and extend those in $[31,33,38,40,42]$. Our results motivate to deal with other types of diffusion problems, for example, reaction diffusion problems, advection-reaction diffusion problems, and nonautonomous reaction diffusion problems.

On the other hand, more general partial differential equations (PDEs) and systems can be considered, for example, system of coupled PDEs, nonlinear diffusion PDEs, and nonautonomous reaction diffusion PDEs. Those kinds of PDEs appear widely as epidemiological models to study and analyze the spread of diseases and pandemics [22-25].

\section{Data Availability}

No data were used to support the study.

\section{Conflicts of Interest}

The authors declare that they have no conflicts of interest.

\section{Acknowledgments}

The authors thank the editors and the referees for their valuable comments and suggestions which improve the quality of the paper. This project was supported by the Academy of Scientific Research and Technology (ASRT), Egypt (Grant No. 6407).

\section{References}

[1] R. P. Agarwal, S. Gala, and M. A. Ragusa, "A regularity criterion in weak spaces to Boussinesq equations," Mathematics, vol. 8, no. 6, p. 920, 2020.

[2] R. P. Agarwal, O. Bazighifan, and M. A. Ragusa, "Nonlinear neutral delay differential equations of fourth-order: oscillation of solutions," Entropy, vol. 23, no. 2, p. 129, 2021.

[3] M. Goltz and J. Huang, Analytical Modeling of Solute Transport in Groundwater, Wiley Online Library, 2017.

[4] M. Hou, X.-X. Xi, and X.-F. Zhou, "Boundary control of a fractional reaction-diffusion equation coupled with fractional ordinary differential equations with delay," Applied Mathematics and Computation, vol. 406, article 126260, 2021.

[5] F. J. Leij, M. T. van Genuchten, and J. Dane, "Mathematical analysis of one-dimensional solute transport in a layered soil profile," Soil Science Society of America Journal, vol. 55, no. 4, pp. 944-953, 1991.

[6] C. Liu and W. P. Ball, "Analytical modeling of diffusionlimited contamination and decontamination in a two-layer porous medium," Advances in Water Resources, vol. 21, no. 4, pp. 297-313, 1998.

[7] X. Liu and M. Jia, "Solvability and numerical simulations for BVPs of fractional coupled systems involving left and right fractional derivatives," Applied Mathematics and Computation, vol. 353, pp. 230-242, 2019.

[8] M. A. Ragusa and F. Wu, "Global regularity and stability of solutions to the $3 \mathrm{~d}$ double-diffusive convection system with Navier boundary conditions," Advances in Differential Equations, vol. 26, no. 7-8, pp. 281-304, 2021.

[9] M. A. Ragusa and F. Wu, "A regularity criterion for threedimensional micropolar fluid equations in Besov spaces of negative regular indices," Analysis and Mathematical Physics, vol. 10, no. 3, pp. 1-11, 2020.

[10] M. T. Van Genuchten, Analytical solutions of the onedimensional convective dispersive solute transport equation, no. 1661, US Department of Agriculture, Agricultural Research Service, 1982.

[11] G. Liu and B. C. Si, "Multi-layer diffusion model and error analysis applied to chamber-based gas fluxes measurements," Agricultural and Forest Meteorology, vol. 149, no. 1, pp. 169178, 2009.

[12] G. Liu and B. C. Si, "Analytical modeling of one-dimensional diffusion in layered systems with position-dependent diffusion coefficients," Advances in Water Resources, vol. 31, no. 2, pp. 251-268, 2008.

[13] S. McGinty, S. McKee, R. M. Wadsworth, and C. McCormick, "Modelling drug-eluting stents," Mathematical Medicine and Biology, vol. 28, no. 1, pp. 1-29, 2011.

[14] G. Pontrelli and F. de Monte, "Mass diffusion through twolayer porous media: an application to the drug-eluting stent," International Journal of Heat and Mass Transfer, vol. 50, no. 17-18, pp. 3658-3669, 2007.

[15] W. J. Addicks, G. Flynn, N. Weiner, and R. Curl, "A mathematical model to describe drug release from thin topical applications," International Journal of Pharmaceutics, vol. 56, no. 3, pp. 243-248, 1989. 
[16] L. Simon and N. Loney, "An analytical solution for percutaneous drug absorption: application and removal of the vehicle," Mathematical Biosciences, vol. 197, no. 2, pp. 119-139, 2005.

[17] R. Pasupuleti, Y. Wang, I. Shabalin, L. Li, Z. Liu, and S. Grove, "Modelling of moisture diffusion in multilayer woven fabric composites," Computational Materials Science, vol. 50, no. 5, pp. 1675-1680, 2011.

[18] S. Mitragotri, Y. G. Anissimov, A. L. Bunge et al., "Mathematical models of skin permeability: an overview," International Journal of Pharmaceutics, vol. 418, no. 1, pp. 115-129, 2011.

[19] J. Caunce, S. Barry, and G. Mercer, "A spatially dependent model for washing wool," Applied Mathematical Modelling, vol. 32, no. 4, pp. 389-404, 2008.

[20] S. Boulaaras, A. Choucha, D. Ouchenane, A. Alharbi, and M. Abdalla, "Result of local existence of solutions of nonlocal viscoelastic system with respect to the nonlinearity of source terms," Fractals, no. article 2240027, 2021.

[21] A. Choucha, D. Ouchenane, S. M. Boulaaras, B. B. Cherif, and M. Abdalla, "Well-posedness and stability result of the nonlinear thermodiffusion full von Kármán beam with thermal effect and time-varying delay," Journal of Function Spaces, vol. 2021, Article ID 9974034, 16 pages, 2021.

[22] E. Raimúndez, E. Dudkin, J. Vanhoefer et al., "Covid-19 outbreak in Wuhan demonstrates the limitations of publicly available case numbers for epidemiological modeling," Epidemics, vol. 34, article 100439, 2021.

[23] A. Viguerie, G. Lorenzo, F. Auricchio et al., "Simulating the spread of COVID-19 via a spatially-resolved susceptibleexposed-infected-recovered-deceased (SEIRD) model with heterogeneous diffusion," Applied Mathematics Letters, vol. 111, article 106617, 2021.

[24] B. Du, X. Lian, and X. Cheng, "Partial differential equation modeling with Dirichlet boundary conditions on social networks," Boundary Value Problems, vol. 2018, Article ID 50, 2018.

[25] R. Hickson, S. Barry, and G. Mercer, "Critical times in multilayer diffusion. Part 1: exact solutions," International Journal of Heat and Mass Transfer, vol. 52, no. 25-26, pp. 57765783, 2009.

[26] E. Tohidi and A. K1lıçman, "An efficient spectral approximation for solving several types of parabolic PDEs with nonlocal boundary conditions," Mathematical Problems in Engineering, vol. 2014, Article ID 369029, 6 pages, 2014.

[27] B. Zogheib and E. Tohidi, "A new matrix method for solving two-dimensional time-dependent diffusion equations with Dirichlet boundary conditions," Applied Mathematics and Computation, vol. 291, pp. 1-13, 2016.

[28] E. Tohidi and F. Toutounian, "Numerical solution of timedependent diffusion equations with nonlocal boundary conditions via a fast matrix approach," Journal of the Egyptian Mathematical Society, vol. 24, no. 1, pp. 86-91, 2016.

[29] B. Zogheib and E. Tohidi, "An accurate space-time pseudospectral method for solving nonlinear multi-dimensional heat transfer problems," Mediterranean Journal of Mathematics, vol. 14, no. 1, p. 30, 2017.

[30] S. Hosseinpour, A. Nazemi, and E. Tohidi, "A new approach for solving a class of delay fractional partial differential equations," Mediterranean Journal of Mathematics, vol. 15, no. 6, pp. 1-20, 2018.

[31] E. Carr and I. Turner, "A semi-analytical solution for multilayer diffusion in a composite medium consisting of a large number of layers," Applied Mathematical Modelling, vol. 40, no. 15-16, pp. 7034-7050, 2016.

[32] E. J. Carr, "New semi-analytical solutions for advection-dispersion equations in multilayer porous media," Transport in Porous Media, vol. 135, no. 1, pp. 39-58, 2020.

[33] E. J. Carr and N. G. March, "Semi-analytical solution of multilayer diffusion problems with time-varying boundary conditions and general interface conditions," Applied Mathematics and Computation, vol. 333, pp. 286-303, 2018.

[34] E. J. Carr and G. Pontrelli, "Modelling mass diffusion for a multi-layer sphere immersed in a semi-infinite medium: application to drug delivery," Mathematical Biosciences, vol. 303, pp. 1-9, 2018.

[35] F. De Monte, "An analytic approach to the unsteady heat conduction processes in one- dimensional composite media," International Journal of Heat and Mass Transfer, vol. 45, no. 6, pp. 1333-1343, 2002.

[36] J. S. Pérez Guerrero, L. C. G. Pimentel, and T. H. Skaggs, “Analytical solution for the advection-dispersion transport equation in layered media," International Journal of Heat and Mass Transfer, vol. 56, no. 1-2, pp. 274-282, 2013.

[37] M. D. Mikhailov and M. N. Ozisik, Unified Analysis and Solutions of Heat and Mass Diffusion, John Wiley \& Sons, New York, 1984.

[38] M. R. Rodrigo and A. L. Worthy, "Solution of multilayer diffusion problems via the Laplace transform," Journal of Mathematical Analysis and Applications, vol. 444, no. 1, pp. 475$502,2016$.

[39] Y. Sun and I. S. Wichman, "On transient heat conduction in a one-dimensional composite slab," International Journal of Heat and Mass Transfer, vol. 47, no. 6-7, pp. 1555-1559, 2004.

[40] R. A. Zimmerman, T. A. Jankowski, and D. M. Tartakovsky, "Analytical models of axisymmetric reaction-diffusion phenomena in composite media," International Journal of Heat and Mass Transfer, vol. 99, pp. 425-431, 2016.

[41] J.-S. Chen, S.-Y. Hsu, M.-H. Li, and C.-W. Liu, "Assessing the performance of a permeable reactive barrier-aquifer system using a dual-domain solute transport model," Journal of Hydrology, vol. 543, pp. 849-860, 2016.

[42] E. Park and H. Zhan, "One-dimensional solute transport in a permeable reactive barrier-aquifer system," Water Resources Research, vol. 45, no. 7, 2009.

[43] R. M. Cotta, "Benchmark results in computational heat and fluid flow: the integral transform method," International Journal of Heat and Mass Transfer, vol. 37, pp. 381-393, 1994.

[44] R. M. Cotta, Integral Transforms in Computational Heat and Fluid Flow, CRC Press, 2020.

[45] R. M. Cotta, D. C. Knupp, C. P. Naveira-Cotta, L. Sphaier, and J. N. Quaresma, "The unified integral transforms (unit) algorithm with total and partial transformation," Computational Thermal Sciences: An International Journal, vol. 6, no. 6, pp. 507-524, 2014.

[46] R. M. Cotta, D. C. Knupp, C. P. Naveira-Cotta, L. A. Sphaier, and J. N. Quaresma, "Unified integral transforms algorithm for solving multidimensional nonlinear convection-diffusion problems," Numerical Heat Transfer, Part A: Applications, vol. 63, no. 11, pp. 840-866, 2013.

[47] L. Sphaier, R. Cotta, C. Naveira-Cotta, and J. Quaresma, “The unit algorithm for solving one-dimensional convectiondiffusion problems via integral transforms," International 
Communications in Heat and Mass Transfer, vol. 38, no. 5, pp. 565-571, 2011.

[48] H. M. Srivastava, M. Luo, and R. K. Raina, "A new integral transform and its applications," Acta Mathematica Scientia, vol. 35, no. 6, pp. 1386-1400, 2015.

[49] F. B. M. Belgacem and R. Silambarasan, "Theory of natural transform," Mathematics in Engineering, Science and Aerospace (MESA) Journal, vol. 3, no. 1, pp. 99-124, 2012.

[50] R. Silambarasn and F. Belgacem, "Applications of the natural transform to Maxwell's equations," Progress In Electromagnetics Research, vol. 899, 2011.

[51] F. B. M. Belgacem and A. A. Karaballi, "Sumudu transform fundamental properties investigations and applications," International Journal of Stochastic Analysis, vol. 2006, Article ID 91083, 23 pages, 2006.

[52] F. B. M. Belgacem, A. A. Karaballi, and S. L. Kalla, "Analytical investigations of the Sumudu transform and applications to integral production equations," Mathematical Problems in Engineering, vol. 2003, no. 3, p. 118, 2003.

[53] Q. D. Katatbeh and F. B. M. Belgacem, "Applications of the Sumudu transform to fractional differential equations," Nonlinear Studies, vol. 18, no. 1, pp. 99-112, 2011.

[54] S. Lang, Complex Analysis, vol. 103, Springer Science \& Business Media, 2013.

[55] R. Haberman, Applied partial differential equations with Fourier series and boundary value problems, Pearson Higher Ed, 2012. 